\title{
Mechanistic Studies of UV Assisted [4+2] Cycloadditions in Synthetic Efforts Toward Vibsanin E
}

Joachim Nikolai, Øystein Loe, Paulina M. Dominiak, ${ }^{a}$ Oksana O. Gerlitz, ${ }^{a}$ Jochen Autschbach $^{*}$ and Huw M. L. Davies ${ }^{*}$

Department of Chemistry, University at Buffalo, The State University of New York, Buffalo, New York, 14260-3000 hdavies@buffalo.edu

\section{Supporting Information}

\section{Contents}

Title of the paper, authors' names, address and table contents.

General information

Experimental procedures and physical data for 16a,b, 17, 19a,b, 24,

25a-c, 27.

Stereochemical assignment for 16a,b, 17, 19a,b, 25a,b.

ORTEP-plot of 27

B3LYP/6-31G(d) optimized structures for trans 24a,b

Selected bond lengths for calculated transition states TS19a-d,

TS20a,b, TS16a-d, TS22a,b, TS11, TS12.

Selected bond lengths for acrolein (triplet) and cycloheptenone (triplet).

Cartesian coordinates, electronic energies and zero point energies for

calculated structures cis 18, trans 18, 14, TS19a-d, TS20a,b, cis $\mathbf{9}$,

trans 9a,b, TS16a-d, TS22a,b, TS11, TS12, 23a,b, cis 24, trans 24a,b,

26a,b, acrolein (triplet), cycloheptenone (triplet).

Complete Ref

${ }^{1} \mathrm{H}$ NMR spectra for 16a,b, 17, 19a,b, 24, 25a-c, 27.
S39

Page Number

S1

S2

S2-6

S6-9

S9

S9

S10

S11

S12-38

S40-49

${ }^{\text {a }}$ Contributions were the X-ray structure determinations 


\section{General Information.}

Commercial reagents were purchased at the highest commercial purity and used without further purification unless otherwise noted. All air and moisture sensitive reactions were performed using glassware flame dried under vacuum and purged with argon prior to use. Tetahydrofuran, hexanes and dichloromethane were dried by passage through a solvent purification system packed with $\mathrm{Al}_{2} \mathrm{O}_{3}$. All other solvents were of reagent grade. Solvents used for rhodium(II)-catalyzed carbenoid transformations were degassed with argon for 10-15 min. prior to use. Analytical thin layer chromatography was performed on $250 \mu \mathrm{m}$ Whatman silica gel (Aluminum backing, UV $254 \mathrm{~nm}$ ) plates using UV light or 50\% sulfuric acid in methanol as visualizing agents. Column chromatography was carried out using E. Merck silica 60 (230 - 400 mesh) or ICN 60 (32 - 64 mesh) following the method of Still. ${ }^{\mathrm{b}}$ Commercially available 1-methoxy-1,3-butadiene was purchased as a mixture of cis and trans isomers. ${ }^{1} \mathrm{H}$ NMR analysis showed a cis/trans ratio of 1:20 favoring the trans isomer. Photochemical reactions were performed by using a highpressure mercury lamp and photolysis vessel (pyrex glass).

NMR spectra were recorded at $293 \mathrm{~K}$ at 400 or $500 \mathrm{MHz}$ for ${ }^{1} \mathrm{H}$ and $75 \mathrm{MHz}$ or $125 \mathrm{MHz}$ for ${ }^{13} \mathrm{C}$. Samples in $\mathrm{CDCl}_{3}$ were calibrated on the solvent peak at $7.27 \mathrm{ppm}$ for the ${ }^{1} \mathrm{H}$ NMR spectra; for ${ }^{13} \mathrm{C}$ NMR spectra the middle solvent peak was set at $77.0 \mathrm{ppm}$. Samples in $\mathrm{C}_{6} \mathrm{D}_{6}$ were calibrated on $0 \mathrm{ppm}$ with TMS as internal standard or on the solvent peak at $7.16 \mathrm{ppm} .{ }^{13} \mathrm{C}$ NMR spectra were set at $0 \mathrm{ppm}$ with TMS or at $128.0 \mathrm{ppm}$ for the middle peak. The following abbreviations are used to explain the multiplicities: $s$, singlet; d, doublet; t, triplet; q, quartet; dd, doublet of doublets; dt, doublet of triplets; ddd, doublet of doublet of doublets. Infrared spectra were recorded on a FT-IR spectrometer.

Experimental Procedures and Physical Data for 16a,b, 17, 19a,b, 24, 25a-c, 27.

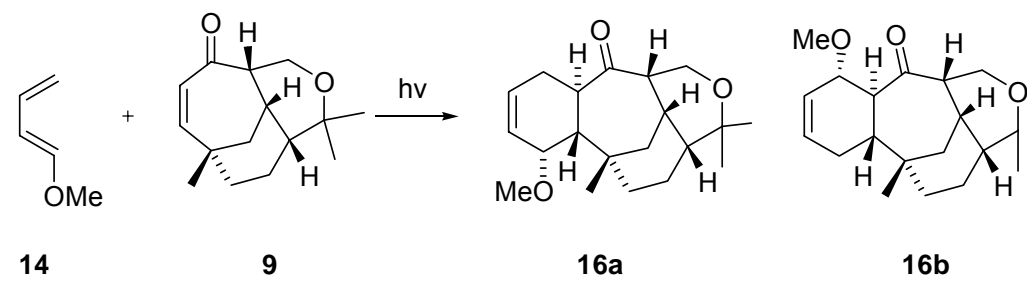

Enone 9 (301 mg, $1.29 \mathrm{mmol}$ ) was dissolved in 1-methoxy-1,3-butadiene (14) (3.0 g, 37 $\mathrm{mmol})$ and dichloromethane was added $(3.0 \mathrm{~mL})$. The reaction mixture was irradiated for $26 \mathrm{~h}$ at which point no starting material was observed by TLC. Excess diene and the dichloromethane were evaporated under reduced pressure and the reaction mixture was purified on a silica gel column with $15 \%$ acetone in hexanes as eluent to afford 16a (158 $\mathrm{mg}, 0.50 \mathrm{mmol}, 39 \%$ ) and $\mathbf{1 6 b}$ (141 $\mathrm{mg}, 0.44 \mathrm{mmol}$, 34\% yield) as well as mixed fraction (110 mg, $0.35 \mathrm{mmol}, 27 \%)$. Crystals of 16a suitable for single crystal X-ray analysis were obtained by recrystallization from diethyl ether. According to a ${ }^{1} \mathrm{H}$ NMR spectrum of the crude reaction mixture $\mathbf{1 6 a}: \mathbf{1 6 b}$ were formed in a $4: 1$ ratio.

16a: ${ }^{1} \mathrm{H}$ NMR (500 MHz, $\left.\mathrm{CDCl}_{3}\right) \delta 5.98(\mathrm{~m}, 1 \mathrm{H}), 5.88(\mathrm{~m}, 1 \mathrm{H}), 4.33$ (dd, $1 \mathrm{H}, J=1.5$, $11.0 \mathrm{~Hz}$ ), 3.85 (dd, 1H, $J=3.5,4.5 \mathrm{~Hz}$ ), 3.51 (dd, $1 \mathrm{H}, J=2.5,11 \mathrm{~Hz}$ ), 3.30 (s, 3H), 3.16

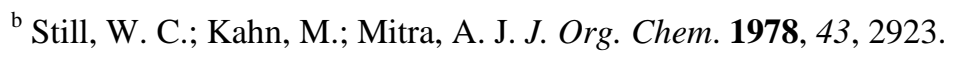


(ddd, 1H, J=5.0, 12, 17) 2.56 (m, 3H), 2.27 (m, 1H), 1.88 (m, 2H), 1.72 (dd, 1H, J=4.5, $13.5 \mathrm{~Hz}$ ), 1.65 (d, broad, $1 \mathrm{H}, J=3.0 \mathrm{~Hz}$ ), 1.46 (dd, $1 \mathrm{H}, J=2.5,12 \mathrm{~Hz}$ ), 1.42 (overlapping dd, $1 \mathrm{H}, J=2.0 \mathrm{~Hz}), 1.26$ (s, 3H), 1.17 (m, 2H), 1.06 (s, 3H), 1.04 (s, 3H) ${ }^{13} \mathrm{C}$ NMR (125 MHz, $\left.\mathrm{CDCl}_{3}\right) \delta 214.2,130.9,126.0,75.0,73.6,62.3,55.2,51.0,50.3,49.2,47.2,44.2,35.8$, 34.0, 33.9, 33.8, 32.5, 27.4, 23.9, 21.7 IR (neat, $\mathrm{cm}^{-1}$ ) 3024, 2967, 2891, 1684, 1462, 1168, 1092 IR (neat, $\mathrm{cm}^{-1}$ ) HRMS (ESI) calcd. for $\mathrm{C}_{20} \mathrm{H}_{30} \mathrm{O}_{3} \mathrm{Na} 341.2087$ found 341.2081

16b: ${ }^{1} \mathrm{H}$ NMR (500 MHz, $\left.\mathrm{C}_{6} \mathrm{D}_{6}\right) \delta 5.86(\mathrm{dd}, 1 \mathrm{H}, J=1.2,10.4 \mathrm{~Hz}), 5.62(\mathrm{~m}, 1 \mathrm{H}), 4.70$ (dddd, $1 \mathrm{H}, J=1.8,3.7,5.5,8.9 \mathrm{~Hz}$ ), 4.06 (dd, $1 \mathrm{H}, J=3.7,12.8 \mathrm{~Hz}$ ), 3.82 (dd, $1 \mathrm{H}, J=$ 7.9, $12.8 \mathrm{~Hz}$ ), 3.43 (dd, 1H, $J=8.9,12.8 \mathrm{~Hz}$ ), 3.22 (s, 3H), 2.79 (m, 1H), 2.14 (m, 1H), 1.90 (m, 1H), 1.79 (m, 1H), $1.70-1.50$ (m, 4H), 1.26 (m, 1H), 1.17 (dd, $1 \mathrm{H}, J=6.7,14.6$ Hz), 1.04 (s, 3H), 0.97 (s, 3H), 0.93 (dd, 1H, $J=6.4,12.8 \mathrm{~Hz}$ ), 0.66 (s, 3H), 0.61 (dd, 1H, $J=4.0,14.0 \mathrm{~Hz}),{ }^{13} \mathrm{C} \mathrm{NMR}\left(75 \mathrm{MHz}, \mathrm{C}_{6} \mathrm{D}_{6}\right)$ 211.6, 127.1, 126.6, 78.1, 72.7, 58.8, 56.4, 54.5, 51.2, 45.0, 42.1, 39.6, 35.4, 35.1, 32.7, 27.3, 26.9, 24,8, 24,6, 23.2

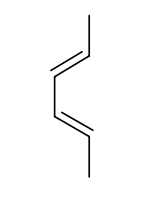

$95.4 \% E / E$

15

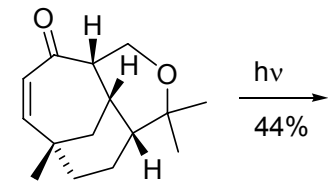

9

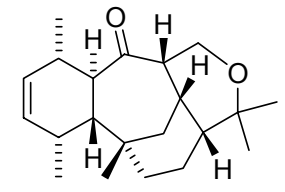

17

Enone 9 (64 mg, $0.29 \mathrm{mmol}$ ) and trans-trans-hexadiene (95.4\% trans-trans, $200 \mathrm{mg}, 2.44$ mmol) were stirred at room temperature and irradiated for $28 \mathrm{hrs}$. The excess diene was evaporated under reduced pressure and the crude product was purified on a silica gel column with $10 \%$ acetone in hexanes as eluent to afford 17 (41 mg, $0.13 \mathrm{mmol}, 45 \%) .{ }^{1} \mathrm{H}$ NMR (500 MHz, $\mathrm{CDCl}_{3}$ ) $\delta 5.59$ (ddd, 1H, $J=2.0,5.5,10.0 \mathrm{~Hz}$ ), 5.36 (d, 1H, $J=10.0$ $\mathrm{Hz}$ ), 4.23 (d, 1H, $J=11.0 \mathrm{~Hz}$ ), 3.57 (dd, 1H, $J=2.4,11.0 \mathrm{~Hz}$ ), 2.52 (m, 3H), 2.36 (dd, $1 \mathrm{H} J=4.4,2.4 \mathrm{~Hz}$ ), 2.18 (dq, 1H, $J=3.9,7.5 \mathrm{~Hz}$ ), 2.07 (ddd, 1H, $J=2.4,4.4,14.7 \mathrm{~Hz}$ ), 1.91 (ddd, $1 \mathrm{H}, J=2.9,5.4,13.7$ ), 1.80 (dd, 1H, $J=3.9,11.2 \mathrm{~Hz}$ ), 1.70 (dd, $1 \mathrm{H}, J=4.0$, $13.7 \mathrm{~Hz}$ ), 1.42 (m, 2H), 1.28 (s, 3H), 1.19 (m, 2H), 1.10 (s, 3H), 1.05 (s, 3H,), 1.02 (d, $3 \mathrm{H}, J=7.0 \mathrm{~Hz}$ ), 0.95 (d, 3H, $J=7.5 \mathrm{~Hz}){ }^{13} \mathrm{C} \mathrm{NMR}\left(125 \mathrm{MHz}, \mathrm{CDCl}_{3}\right) \delta 212.8,133.7$, 131.4, 73.5, 62.4, 55.4, 51.8, 50.2, 48.4, 43.9, 39.3, 34.2, 33.7, 33.4, 33.2, 32.8, 27.3, 24.0, 21.2, 20.8, 16.1 IR (neat, $\mathrm{cm}^{-1}$ )2970, 2900, 1683, 1455, 1377, 1161, 1094, 737 HRMS (ESI) calcd. for $\mathrm{C}_{21} \mathrm{H}_{32} \mathrm{O}_{2} \mathrm{Na} 339.2295$ found 339.2292.

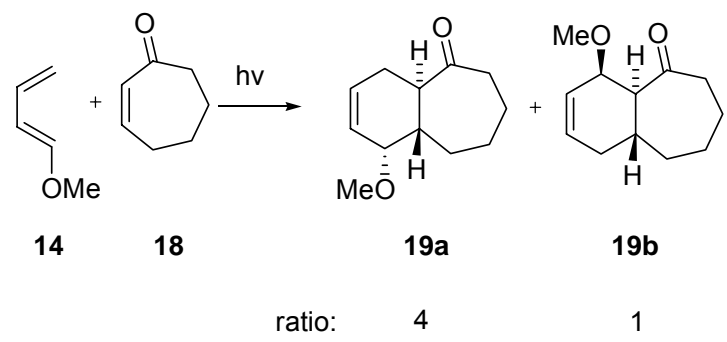

Cyclohepteneone (18) (90 mg, $0.82 \mathrm{mmol}$ ) was dissolved in 1-methoxy-1,3-butadiene (14) (1.40 g, $16.7 \mathrm{mmol})$ were stirred at room temperature and irradiated for $24 \mathrm{~h}$. The reaction was monitored by TLC. After complete conversion of 18 excess 1-methoxy-1,3- 
butadiene was removed under reduced pressure. The reaction mixture was purified by column chromatography with $5 \%$ acetone in dichloromethane as eluent to afford 19a,b (149 mg, $0.77 \mathrm{mmol}, 94 \%$ ). According to a ${ }^{1} \mathrm{H}$ NMR spectrum of the crude reaction mixture 19a:19b were formed in a 3:1 ratio.

19a: ${ }^{1} \mathrm{H}$ NMR (500 MHz, $\mathrm{CDCl}_{3}$ ) $\delta 6.02$ (m, 1H), 5.94 (ddd, $1 \mathrm{H}, J=2.4,4.9,10.1 \mathrm{~Hz}$ ), 3.55 (dd, 1H, $J=3.6,3.6 \mathrm{~Hz}$ ), 3.39 (s, 3H), 2.73 (ddd, 1H, $J=5.0,5.0,11.5 \mathrm{~Hz}$ ), 2.63 (ddd, 1H, $J=4.0,4.0,11.0 \mathrm{~Hz}$ ), 2.42 (m, 1H), 2.21 (ddd, 1H, $J=1.5,5.5,18.5 \mathrm{~Hz}$ ), 2.09 - 1.91 (m, 3H), 1.82 (dd, $1 \mathrm{H}, J=6.1,14.6 \mathrm{~Hz}), 1.66-1.40(\mathrm{~m}, 4 \mathrm{H}) .{ }^{13} \mathrm{C}$ NMR $(75 \mathrm{MHz}$, $\left.\mathrm{CDCl}_{3}\right) \delta 215.8,130.2,126.2$, 76.4, 57.1, 48.3, 42.0, 41.0, 31.8, 29.6, 28.8, 25.7. HRMS (EI) calcd. for $\mathrm{C}_{12} \mathrm{H}_{18} \mathrm{O}_{2} 194.1301$ found 194.1304.

19b: ${ }^{1} \mathrm{H}$ NMR (500 MHz, $\mathrm{C}_{6} \mathrm{D}_{6}$ ) $\delta 5.76$ (dddd, $1 \mathrm{H}, J=1.5,2.3,4.6,10.0 \mathrm{~Hz}$ ), 5.65 (ddd, $1 \mathrm{H}, J=1.8,5.2,10.0 \mathrm{~Hz}$ ), 3.91 (dd, $1 \mathrm{H}, J=3.5,4.6 \mathrm{~Hz}$ ), 3.04 (s, 3H), 2.73 (ddd, $1 \mathrm{H}, J=$ 2.1, 11.3, $13.4 \mathrm{~Hz}$ ), 2.57 (dd, 1H, $J=7.0,11.3 \mathrm{~Hz}$ ), 2.08 (dd, 1H, $J=4.0,11.9 \mathrm{~Hz}$ ), 2.02 (m, 1H), 1.80 (ddd, 1H, J = 1.2, 5.2, $18.3 \mathrm{~Hz}$ ), 1.59 (m, 1H), 1.52 (m, 1H), 1.37 - 1.31 $(\mathrm{m}, 2 \mathrm{H}), 1.27-1.12(\mathrm{~m}, 2 \mathrm{H}), 0.80(\mathrm{~m}, 1 \mathrm{H}) .{ }^{13} \mathrm{C} \mathrm{NMR}\left(75 \mathrm{MHz}, \mathrm{CDCl}_{3}\right) \delta 216.9,131.9$, 124.9, 76.6, 59.6, 57.1, 45.0, 36.5, 34.3, 31.0, 30.1, 26.6. HRMS (EI) calcd. for $\mathrm{C}_{12} \mathrm{H}_{18} \mathrm{O}_{2}$ 194.1301 found 194.1301

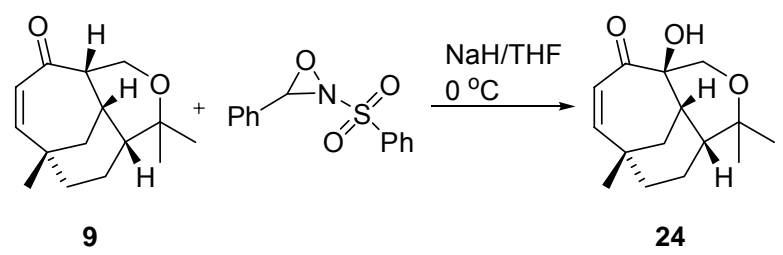

Enone 9 (100 mg, $0.427 \mathrm{mmol})$ was dissolved in THF (8 mL) at $0{ }^{\circ} \mathrm{C}$ and $\mathrm{NaH}(52 \mathrm{mg}$, $2.2 \mathrm{mmol}$ ) was added. The reaction was stirred for $20 \mathrm{~min}$ and Davis' oxaziridine ${ }^{\mathrm{c}}$ (160 $\mathrm{mg}, 0.61 \mathrm{mmol})$ in THF (5 mL) was added. The reaction mixture was kept at the same temperature and stirred for 3 hours. The mixture was diluted with ether $(50 \mathrm{ml})$ and extracted with water $(100 \mathrm{~mL})$ and then brine $(50 \mathrm{~mL})$. The organics were dried over magnesium sulfate, filtered and concentrated under reduced pressure. The crude product was purified by column chromatography on silica gel with $10 \%$ acetone in dichloromethane as the eluent to afford 24 (57 mg, $0.23 \mathrm{mmol}, 53 \%)$.

${ }^{1} \mathrm{H} \mathrm{NMR}\left(500 \mathrm{MHz}, \mathrm{CDCl}_{3}\right) \delta 6.15$ (dd, $\left.1 \mathrm{H}, J=1.5,12.5 \mathrm{~Hz}\right), 6.07$ (d, $1 \mathrm{H}, J=12.5 \mathrm{~Hz}$ ), 4.09 (d, 1H, $J=11.8 \mathrm{~Hz}$ ), 3.28 (d, 1H, $J=11.8 \mathrm{~Hz}$ ), 3.04 (s, 1H), 2.52 (s $1 \mathrm{H}), 2.27$ (d, $1 \mathrm{H}, J=14.3 \mathrm{~Hz}), 1.66-1.62(\mathrm{~m}, 2 \mathrm{H}), 1.55-1.47(\mathrm{~m}, 2 \mathrm{H}), 1.37$ (s, 3H), 1.34 - 1.26 (m, 3H), 1.18 (s, 3H), 1.15 (s, 3H). ${ }^{13} \mathrm{C}$ NMR(125 MHz, $\left.\mathrm{CDCl}_{3}\right) \delta$ 203.3, 153.7, 128.0, 78.6, 74.2, 67.6, 42.6, 37.9, 37.5, 37.3, 36.9, 30.9, 26.6, 26.2, 22.1. IR (neat, $\mathrm{cm}^{-1}$ ) 3393, 2936, 1648, 1457, 1243, 1095, 1064. HRMS (ESI) calcd. for $\mathrm{C}_{15} \mathrm{H}_{22} \mathrm{O}_{3} \mathrm{Na} 273.1461$ found 273.1470

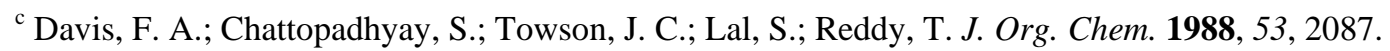




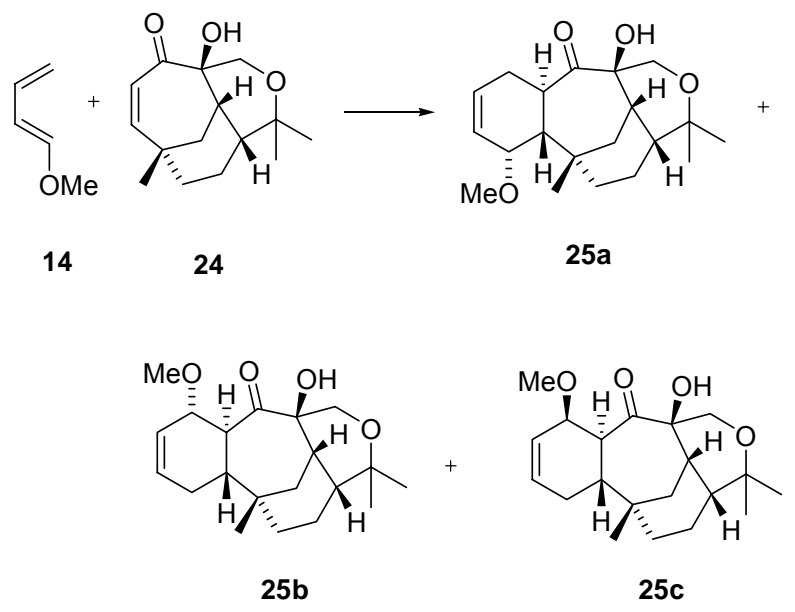

Hydroxy ketone 24 (70 mg, $0.28 \mathrm{mmol}$ ) was dissolved in 1-methoxy-1,3-butadiene (14) $(2.5 \mathrm{~g}, 30 \mathrm{mmol})$ and dichloromethane $(2.5 \mathrm{~mL})$ was added. The reaction was stirred at room temperature and irradiated for $60 \mathrm{~h}$. The progress of the reaction was monitored by TLC. After complete conversion of $\mathbf{2 4}$, excess diene was removed under reduced pressure and the product was purified by column chromatography on silica gel with $12 \%$ acetone in hexanes to afford 25a (12 mg, $0.036 \mathrm{mmol}, 13 \%), 25 \mathrm{~b}$ (12 mg, $0.036 \mathrm{mmol}, 13 \%)$ and 25c (5 mg, $0.015 \mathrm{mmol}, 5 \%$ ) as well as mixed fractions (45 mg, $0.13 \mathrm{mmol}, 48 \%$ ). Samples of analytical purity of 25a-c were obtained by preparative TLC with $3 \%$ methanol in dichloromethane as eluent.

25a: ${ }^{1} \mathrm{H}$ NMR (500 MHz, $\left.\mathrm{C}_{6} \mathrm{D}_{6}\right) \delta 5.89(\mathrm{~m}, 1 \mathrm{H}), 5.67(\mathrm{~m}, 1 \mathrm{H}), 4.51(\mathrm{~s}, 1 \mathrm{H}, \mathrm{OH}), 4.06$ (ddd, $1 \mathrm{H}, J=6.4,7.3,13.1 \mathrm{~Hz}$ ), 3.78 (d, $1 \mathrm{H}, J=12.8 \mathrm{~Hz}$ ), 3.73 (d, $1 \mathrm{H}, J=12.8 \mathrm{~Hz}$ ), 3.54 (dd, $1 \mathrm{H}, J=1.5,5.8 \mathrm{~Hz}$ ), 3.02 (s, 3H), 2.83 (dddd, $1 \mathrm{H}, J=3.8,7.6,18.9 \mathrm{~Hz}) 2.43$ (m, $1 \mathrm{H}$ ), 2.26 - 2.17 (m, 2H), 2.07 (dddd, $1 \mathrm{H}, J=3.0,4.9,7.6,18.9 \mathrm{~Hz}$ ), 1.61 (d, broad, $1 \mathrm{H}, J$ = $14.6 \mathrm{~Hz}), 1.46(\mathrm{~m}, 1 \mathrm{H}), 1.42(\mathrm{~d}$, broad, $1 \mathrm{H}, J=12.8 \mathrm{~Hz}), 1.30(\mathrm{~s}, 3 \mathrm{H}), 1.21-1.11(\mathrm{~m}$, $2 \mathrm{H}), 1.14$ (s, 3H), 0.81 (ddd $1 \mathrm{H}, J=2.7,12.5,13.1 \mathrm{~Hz}), 0.80$ (s, 3H). ${ }^{13} \mathrm{C}$ NMR $(75 \mathrm{MHz}$, $\left.\mathrm{C}_{6} \mathrm{D}_{6}\right) \delta 214.8,130.7,127.3,77.0,73.6,72.6,67.4,54.9,51.8,41.8,41.4,40.1,36.8$, 36.5, 34.8, 33.6, 29.4, 27.0, 24.2, 23.2.

25b: ${ }^{1} \mathrm{H}$ NMR (500 MHz, $\mathrm{C}_{6} \mathrm{D}_{6}$ ) $\delta 5.79$ (dd, broad, $1 \mathrm{H}, J=1.5,10.4 \mathrm{~Hz}$ ), 5.74 (dddd, $1 \mathrm{H}$, $J=2.4,5.2,7.6,10.4 \mathrm{~Hz}$ ), 5.02 (s, $1 \mathrm{H}, \mathrm{OH}), 4.67$ (ddd, $1 \mathrm{H}, J=1.5,5.5,8.8 \mathrm{~Hz}$ ), 4.19 (d, $1 \mathrm{H}, J=12.8 \mathrm{~Hz}), 3.97$ (d, $1 \mathrm{H}, J=12.8 \mathrm{~Hz}), 3.58(1 \mathrm{H}, \mathrm{dd}, J=8.8,13.1 \mathrm{~Hz}), 3.12(\mathrm{~s}, 3 \mathrm{H})$, 2.46 (dd, $1 \mathrm{H}, J=7.0,7.0 \mathrm{~Hz}$ ), $1.88(\mathrm{~m}, 1 \mathrm{H}), 1.75(\mathrm{~m}, 1 \mathrm{H}), 1.65-1.51$ (m, 4H), 1.29 (s, 3H), 1.23 (m, 1H), $1.13-1.07$ (m, 2H), 1.06 (s, 3H), 0.59 (s, 3H), 0.58 (hidden by Me-gr, $1 \mathrm{H}) .{ }^{13} \mathrm{C}$ NMR $\left(75 \mathrm{MHz}, \mathrm{C}_{6} \mathrm{D}_{6}\right) \delta 211.2,125.3,124.0,75.9,74.4,71.0,64.4,54.0,50.5$, 43.3, 38.0, 37.2, 34.0, 33.4, 32.5, 30.1, 25.1, 24.9, 21.8, 20.2.

25c: ${ }^{1} \mathrm{H}$ NMR (500 MHz, $\mathrm{C}_{6} \mathrm{D}_{6}$ ) $\delta$ 5.68-5.61 (m, 2H), 4.77 (s, $\left.1 \mathrm{H}, \mathrm{OH}\right), 4.67(\mathrm{~d}, 1 \mathrm{H}, J=$ $10.6 \mathrm{~Hz}$ ), 4.02 (ddd, $1 \mathrm{H}, J=1.2$, 3.4, $4.6 \mathrm{~Hz}$ ), 3.57 (d, $1 \mathrm{H}, J=10.6 \mathrm{~Hz}$ ), $2.83(\mathrm{~s}, 3 \mathrm{H}$ ), 2.54 - 2.46 (m, 3H), 1.99 (ddd, $1 \mathrm{H}, J=4.3,10.4,12.2 \mathrm{~Hz}$ ), 1.88 (ddd, $1 \mathrm{H}, J=4.3$, 4.6, $18.0 \mathrm{~Hz}$ ), $1.47-1.17$ (m, 4H), 1.19 (s 3H), 1.10 (s 3H), 1.10 (1H, hidden by Me gr), 1.02 (ddd, $1 \mathrm{H}, J=3.7,3.7,13.1 \mathrm{~Hz}$ ), 0.80 (ddd, $1 \mathrm{H}, J=6.1,13.7,14.0 \mathrm{~Hz}$ ), 0.68 (s, $3 \mathrm{H}$ ). ${ }^{13} \mathrm{C}$ NMR (75 MHz, $\left.\mathrm{C}_{6} \mathrm{D}_{6}\right) \delta 207.7,133.5,123.8,78.6,75.7,73.1,67.9,58.7,55.9,44.7$, 43.9, 40.5, 40.1, 33.1, 32.9, 31.2, 30.0, 27.3, 24.0, 22.5. 


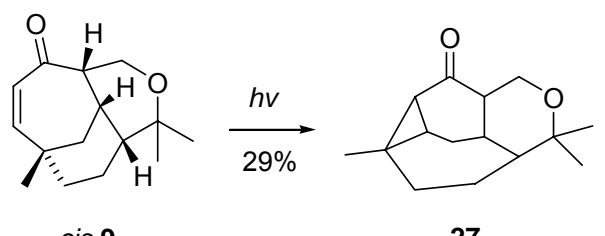

cis 9

27

Enone 9 (45 mg, 0.19mmol) was dissolved in dichloromethane $(2 \mathrm{ml})$ and irradiated under UV light for $48 \mathrm{hrs}$ at room temperature. The reaction was monitored by TLC. After completion, the solvent was removed under reduced pressure and the crude product was purified by coloumn chromatography with 5-25\% EtOAc in hexane as eluent to afford 27 (13 mg, $0.028 \mathrm{mmol}, 29 \%)$. Crystals suitable for single crystal analysis were obtained from recrystallization from diethyl ether.

${ }^{1} \mathrm{H}$ NMR (500 MHz, $\mathrm{CDCl}_{3}$ ) $\delta 4.53$ (d, $1 \mathrm{H}, J=11.9 \mathrm{~Hz}$ ), 3.57 (dd, $1 \mathrm{H}, J=4.3,11.9 \mathrm{~Hz}$ ), 2.74 (dd, $1 \mathrm{H}, J=5.8,5.8 \mathrm{~Hz}$ ), 2.29, (ddd, $1 \mathrm{H}, J=6.1,7.3,14.0$ ), 1.97 (d, $1 \mathrm{H}, J=14.3$ ), $1.95(\mathrm{~m}, 1 \mathrm{H}), 1.90-1.83(\mathrm{~m}, 2 \mathrm{H}), 1.75(\mathrm{~m}, 1 \mathrm{H}), 1.53(\mathrm{~d}, 1 \mathrm{H}, J=7.0 \mathrm{~Hz}), 1.33$ (d, $1 \mathrm{H}, J$ $=6.4,6.4 \mathrm{~Hz}), 1.27(\mathrm{~s}, 3 \mathrm{H}), 1.23-1.18(\mathrm{~m}, 2 \mathrm{H}), 1.17(\mathrm{~s}, 3 \mathrm{H}), 1.08(\mathrm{~s}, 3 \mathrm{H}) .{ }^{13} \mathrm{C}$ NMR $(75$ $\left.\mathrm{MHz}, \mathrm{CDCl}_{3}\right) \delta 208.6,74.5,58.4,47.4,45.1,34.9,33.8,30.3,30.1,29.6,28.4,25.9$, 25.7, 25.2, 23.0. IR (neat $\mathrm{cm}^{-1}$ ) 2941, 1681, 1450, 1378, 1232, 1200, 1145, 1104, 1090, 1021. HRMS (ESI) calcd. for $\mathrm{C}_{30} \mathrm{H}_{44} \mathrm{O}_{4} \mathrm{Na} 491.3132$ found 491.3141

\section{Stereochemical Assignment for 16a,b, 17, 19a,b, 25a,b}

16a,b:

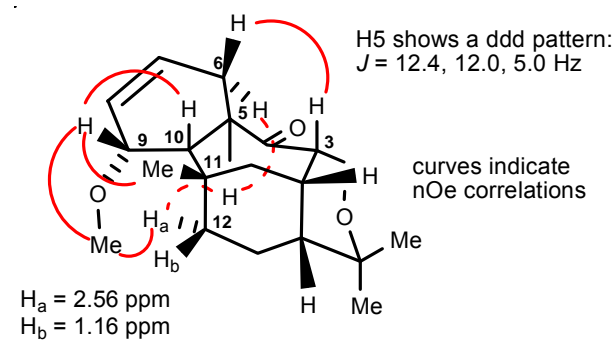

$16 a$

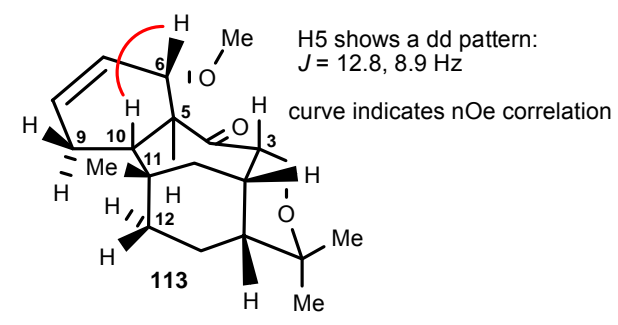

$16 b$

The ${ }^{1} \mathrm{H}$ NMR spectrum of $\mathbf{1 6 a}$ gives rise to a ddd pattern for the hydrogen atom at C5, indicating a methylene unit at C6. The coupling pattern of this hydrogen atom is a critical spectroscopic probe to establish the substitiution pattern at C6 as well as the trans configuration of the $\mathrm{C} 5-\mathrm{C} 10$ bond. For $\mathrm{H} 5$ at $\mathrm{C} 5$ in 16a the three coupling constants were determined as $J=12.4,12.0,5.0 \mathrm{~Hz}$. One large coupling constant can be attributed to an axial-axial interaction with $\mathrm{H} 10$, indicating an anti configuration for $\mathrm{C} 5-\mathrm{C} 10$. The second large coupling constant can be assigned to an axial-axial coupling to the axial hydrogen atom at C6. The small coupling constant $(J=5.0 \mathrm{~Hz})$ is attributed to an axialequatorial between $\mathrm{H} 5$ at $\mathrm{C} 5$ and the equatorial $\mathrm{H6}$ at C6. Furthermore, it is important to 
note that the methoxy group occupies an equatorial position. This is determined from the nOe correlation between the proton at position 10 and the hydrogen atom at C9 (Figure 3). The chemical shift of the hydrogen atom at position 10 was identified by a ${ }^{1} \mathrm{H}$ COSY experiment. More important however, the methoxygroup shows an nOe to the hydrogen atom at position 12. Surprisingly, the resonance of the hydrogen atoms attached to carbon C12 are found at $2.56 \mathrm{ppm}$ and $1.16 \mathrm{ppm}$. The unexpected $2.56 \mathrm{ppm}$ shift for one of the hydrogen atoms is readily understood be realizing that this hydrogen atom is placed right above the anisotropic cone of the carbonyl group of the rather twisted cycloheptenone. An nOe correlation between the proton pointing 'up' at C6 and H10 is expected but both these signals are partially overlapping with other protons and hence an nOe could not be verified. For 16b the hydrogen atom at C5 displays a dd pattern, indicating that the methoxy group is located at position 6 . The relative stereochemistry of the methoxy group was assigned as 'down' as the proton at C5 has two large coupling constants $(J=$ 12.8, $8.9 \mathrm{~Hz}$ ). In addition there is an nOe effect between the hydrogen atom at C6 and the hydrogen atom at C10. The hydrogen atom at C10 is known to be oriented towards the convex face ('up') of the molecule and hence the methoxygroup has to be 'down'.

X-ray crystallographic data for 16a. The structural assignment of 16a using NMR analysis was confirmed by X-ray crystallography. The cystallographic data for 16a are available in the attached CIF file and at the Cambridge Crystallographic Data Center, CCDC 270719 [Dominiak, P. M.; Coppens P. Private Communication].

17:

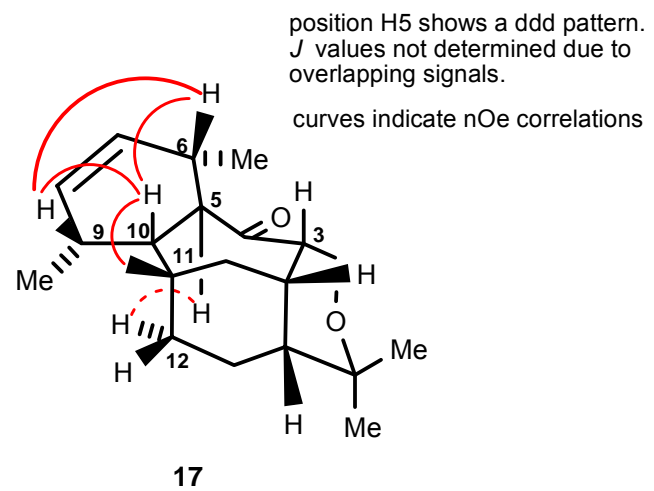

The hydrogen atom at C10 is identified at $1.80 \mathrm{ppm}$ and the hydrogen atom at C9 is found at $2.17 \mathrm{ppm}$. There are nOe interactions between $\mathrm{H} 9$ and the hydrogen atom at C10. This unambigiously assigns the methyl group at C9 to be down. Its signal is found at $0.95 \mathrm{ppm}$. A nOe was also observed between the hydrogen atom at C9 and C6. Therefore, these two protons have to be cis to each other, pointing up and hence the methyl group C6 has to be oriented towards the concave side of $\mathbf{1 7}$. The resonance of the methyl group at C6 is found at $1.03 \mathrm{ppm}$. The signal at C12 shows a nOe to the hydrogen at C5. 
19a,b:

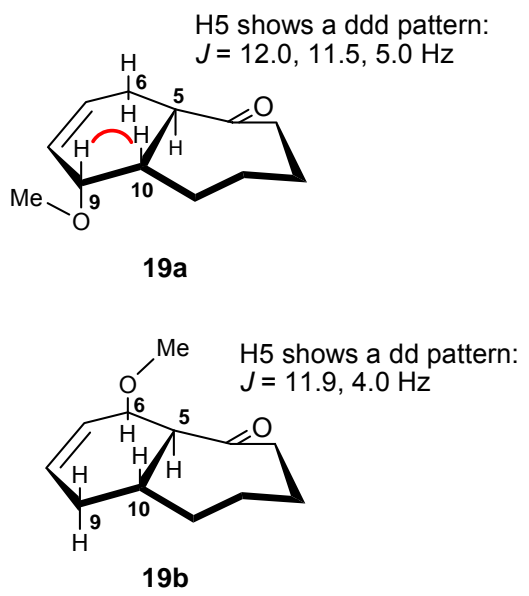

The structures of photoadducts 19a and 19b were assigned by nOe studies and on the basis of characteristic coupling constants. For the major product 19a the methoxy group was determined to be located on C9 as the hydrogen atom attached to C5 shows a ddd pattern $(J=12.0,11.5,5.0 \mathrm{~Hz})$, very similar to the structure 16a. Consequently, this requires a methylene unit at position 6 . The resonance of the hydrogen atom at C10 was identified by a COSY experiment and this hydrogen atom showed a clear nOe effect to the hydrogen atom at C9. No nOe between the methoxy group and the hydrogen atom at C10 was seen, indicating that the methyl ether at position 9 is 'down'. For compound 19b the resonance of the hydrogen atom at C5 is a characteristic dd system $(J=11.9,4.0 \mathrm{~Hz})$ which indicates that the methoxy group is attached to C6. A small coupling constant of $4.0 \mathrm{~Hz}$ indicates that the methyl ether is 'up'

\section{5a,b:}

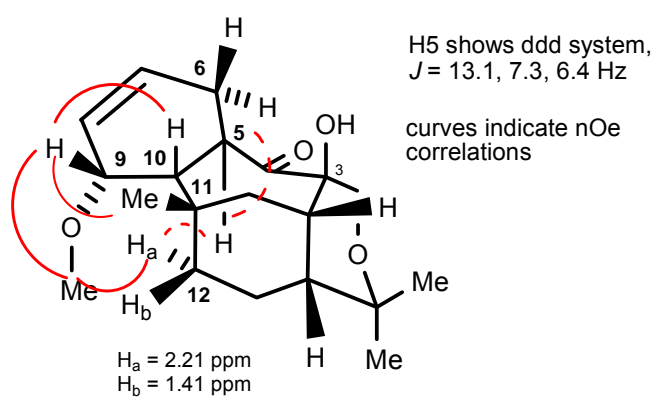

The hydroxy group is located on C9 because H5 shows a ddd pattern $(J=6.4,7.3,13.1$ $\mathrm{Hz}$ ). Compound 25a shows nOe's very similar to 16a. The hydrogen atom at C9 shows an nOe to the hydrogen atom at C10. This H10 is known to be oriented towards the convex side of the molecule. Since the methoxy group shows no effect to this H10 the methyl ether is assigned to be oriented towards the concave face ("down") of the tetracycle. This is supported by the fact that the methyl ether shows an nOe to hydrogen atom at C12. All nOe's observed in 25a are also found for $\mathbf{1 6 a}$. 


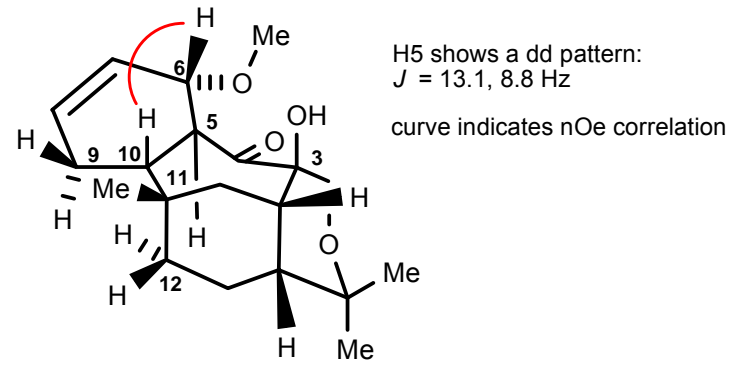

25b

For the hydrogen atom at C5 shows a dd pattern $(J=8.8,13.1 \mathrm{~Hz})$ indicating that the methoxygroup is found on C6. The magnitude of these coupling constants indicates an anti configuration at $\mathrm{C} 5$ and $\mathrm{C} 10$. Furthermore, there is an nOe correlation between the hydrogens at C6 and C10. Therefore, the hydrogen atom located at C5 is oriented towards the convex side of the molecule and hence the methoxy group is located towards the concave side of the tetracycle.

ORTEP-plot of 27. (The cystallographic data for $\mathbf{2 7}$ are available in the attached CIF file and at the Cambridge Crystallographic Data Center, CCDC 270719 [Dominiak, P. M.; Coppens P. Private Communication].

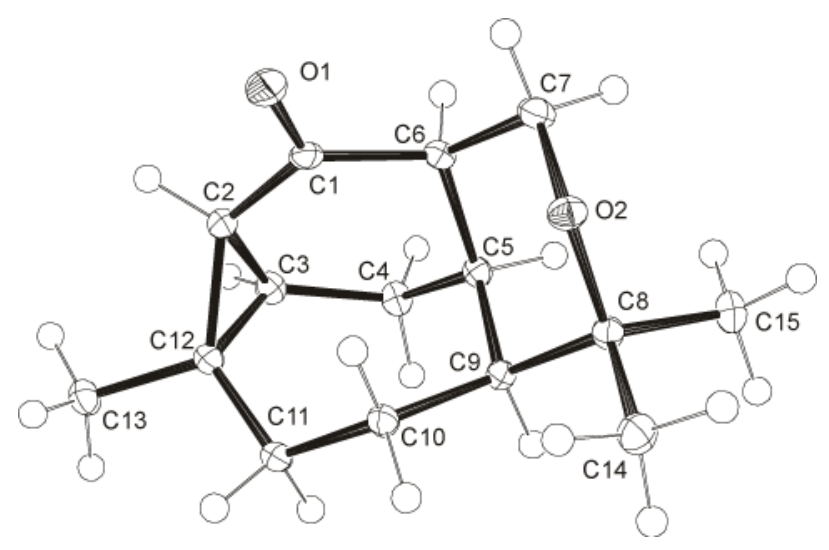

The ellipsoids of thermal vibration represent a 50\% probability.

B3L YP/6-31G(d) optimized structures for trans 24a,b.

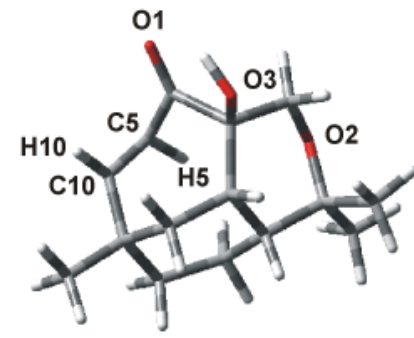

trans 24a

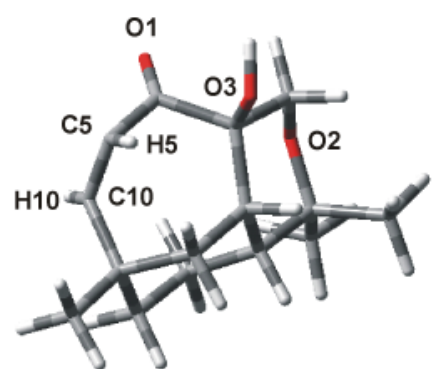

trans 24b 
Selected bond lengths for calculated transition states

\begin{tabular}{l|ccc}
$\begin{array}{l}\text { Transition state } \\
\text { Level of theory }\end{array}$ & TS19a & $\begin{array}{c}\text { TS19b } \\
\text { B3LYP/6-311+G(d,p) }\end{array}$ & $\begin{array}{c}\text { TS19c } \\
\text { B3LYP/6-311+G(d,p) }\end{array}$ \\
\hline C8-C9 $[\AA]$ & 1.36346 & 1.37011 & 1.38016 \\
C7-C8 $[\AA]$ & 1.42003 & 1.41919 & 1.41376 \\
C6-C7 $[\AA]$ & 1.37234 & 1.37612 & 1.37278 \\
C5-C9 $[\AA]$ & - & 2.79828 & 2.42465 \\
C6-C10 $[\AA]$ & - & 2.24327 & 2.33943 \\
C9-C10 $[\AA]$ & 2.71825 & - & - \\
C5-C6 $[\AA]$ & 2.27423 & - & - \\
\hline
\end{tabular}

\begin{tabular}{l|c|c|c}
$\begin{array}{l}\text { Transition state } \\
\text { Level of theory }\end{array}$ & TS19d & $\begin{array}{c}\text { TS20a } \\
\text { B3LYP/6-311+G(d,p) }\end{array}$ & $\begin{array}{c}\text { TS20b } \\
\text { B3LYP/6-311+G(d,p) }\end{array}$ \\
\hline C8-C9 $[\AA]$ & 1.36380 & 1.38461 & 1.39324 \\
C7-C8 $[\AA]$ & 1.41829 & 1.39742 & 1.39330 \\
C6-C7 $[\AA]$ & 1.37309 & 1.40353 & 1.38938 \\
C5-C9 $[\AA]$ & - & 2,63517 & - \\
C6-C10 $[\AA]$ & - & 1.98981 & - \\
C9-C10 $[\AA]$ & 2.75418 & - & 2.22615 \\
C5-C6 $[\AA]$ & 2.24443 & - & 2.21149 \\
\hline
\end{tabular}

\begin{tabular}{|c|c|c|c|}
\hline $\begin{array}{l}\text { Transition state } \\
\text { Level of theory }\end{array}$ & $\begin{array}{c}\text { TS16a } \\
\text { B3LYP/6-31G(d) }\end{array}$ & $\begin{array}{c}\text { TS16b } \\
\text { B3LYP/6-31G(d) }\end{array}$ & $\begin{array}{c}\text { TS16c } \\
\text { B3L } / 6-31 G(d)\end{array}$ \\
\hline C8-C9 $[\AA]$ & 1.36335 & 1.37444 & 1.36352 \\
\hline C7-C8 $[\AA]$ & 1.42578 & 1.41651 & 1.42433 \\
\hline C6-C7 $[\AA]$ & 1.37291 & 1.37130 & 1.37402 \\
\hline C5-C9 $[\AA]$ & - & 2.43657 & - \\
\hline C6-C10 $[\AA]$ & - & 2.49839 & - \\
\hline C9-C10 $[\AA]$ & 2.88338 & - & 2.90455 \\
\hline C5-C6 $[\AA]$ & 2.25188 & - & 2.23198 \\
\hline
\end{tabular}

\begin{tabular}{l|ccc}
$\begin{array}{l}\text { Transition state } \\
\text { Level of theory }\end{array}$ & TS16d & TS22a & TS22b \\
B3-C9 $[\AA]$ & 1.36899 & B3LYP/6-31G(d) & B3LYP/6-31G(d) \\
\hline C7-C8 $[\AA]$ & 1.41943 & 1.38191 & 1.39580 \\
C6-C7 $[\AA]$ & 1.37676 & 1.39796 & 1.39065 \\
C5-C9 $[\AA]$ & 2.84255 & 1.41519 & 1.40682 \\
C6-C10 $[\AA]$ & 2.34450 & 2.87567 & - \\
C9-C10 $[\AA]$ & - & 1.93364 & - \\
C5-C6 $[\AA]$ & - & - & 2.44118 \\
\hline
\end{tabular}

\begin{tabular}{l|cc}
$\begin{array}{l}\text { Transition state } \\
\text { Level of theory }\end{array}$ & TS11 & TS12 \\
\hline C8-C9 $[\AA]$ & B3LYP/6-31G(d) & B3LYP/6-31G(d) \\
C7-C8 $[\AA]$ & 1.37286 & 1.36555 \\
C6-C7 $[\AA]$ & 1.43575 & 1.43510 \\
C5-C9 $[\AA]$ & 1.36122 & 1.36854 \\
C6-C10 $[\AA]$ & 2.29975 & -- \\
C9-C10 $[\AA]$ & 2.69867 & -- \\
C5-C6 $[\AA]$ & -- & 2.68852 \\
\hline
\end{tabular}


Selected bond lengths for acrolein (triplet) and cycloheptenone (triplet)

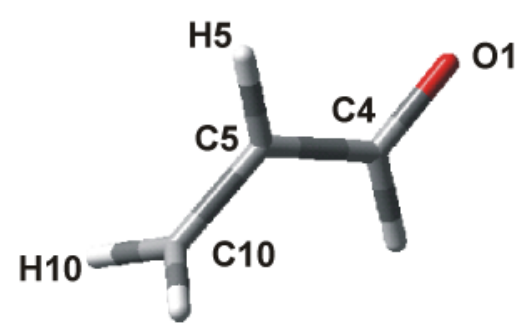

acrolein (triplet)

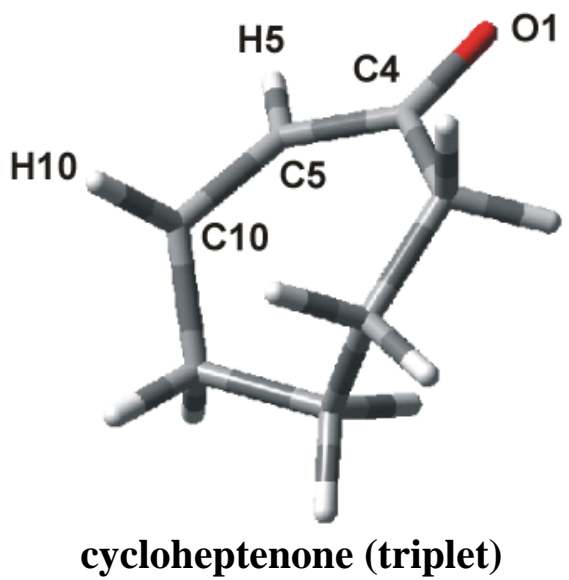

\begin{tabular}{l|cc} 
& acrolein (triplet) & cycloheptenone (triplet) \\
\hline $\mathrm{E}_{\text {tot }}{ }^{a}+\mathrm{ZPE}^{\mathrm{b}}$ & -191.763484 & -347.723996 \\
$<\mathrm{S}^{2}>$ & 2.0172 & 2.0162 \\
$\mathrm{~d}(\mathrm{C} 5-\mathrm{C} 10)^{\mathrm{c}}[\AA]$ & $1.45403(1.33804)$ & $1.45820(1.34519)$ \\
$\mathrm{d}(\mathrm{C} 4-\mathrm{C} 5)^{\mathrm{c}}[\AA]$ & $1.43870(1.47508)$ & $1.44814(1.48570)$ \\
$\mathrm{d}(\mathrm{C} 4-\mathrm{O})^{\mathrm{c}}[\AA]$ & $1.23633(1.21546)$ & $1.24121(1.22556)$ \\
$\mathrm{H} 5-\mathrm{C} 5-\mathrm{C} 10-\mathrm{H} 10\left[^{\circ}\right]$ & 87.065 & 88.046 \\
\hline
\end{tabular}

\footnotetext{
${ }^{a}$ Hartree per molecule

${ }^{\mathrm{b}}$ ZPE: zero point energy in harmonic approximation; unscaled

${ }^{\mathrm{c}}$ bond length for ground state acrolein and cis cycloheptenone (18) in parenthesis. Note that the numbering does not correspond to the systematic nomenclature of these compounds.
} 
Cartesian coordinates, electronic energies and zero point energies for calculated structures

\begin{tabular}{l|ccc} 
& \multicolumn{3}{|c}{ cis 18; B3LYP/6-311+G(d,p) } \\
\hline $\mathrm{C}$ & 0.64067700 & 1.62783500 & -0.01643300 \\
$\mathrm{H}$ & 0.87515600 & 2.69113700 & -0.04898100 \\
$\mathrm{C}$ & -0.66976200 & 1.34195100 & 0.04679200 \\
$\mathrm{H}$ & -1.36302200 & 2.17825600 & 0.06148400 \\
$\mathrm{C}$ & -1.40601600 & 0.05249000 & 0.03426500 \\
$\mathrm{O}$ & -2.62226200 & 0.09540200 & -0.07193200 \\
$\mathrm{C}$ & -0.73207100 & -1.30361500 & 0.19326200 \\
$\mathrm{H}$ & -0.69924200 & -1.49655100 & 1.27475800 \\
$\mathrm{H}$ & -1.43366300 & -2.03038400 & -0.22242300 \\
$\mathrm{C}$ & 0.66985100 & -1.50781900 & -0.38972900 \\
$\mathrm{H}$ & 0.90414800 & -2.57352500 & -0.30405500 \\
$\mathrm{H}$ & 0.67056300 & -1.28407900 & -1.46349500 \\
$\mathrm{C}$ & 1.76722600 & -0.70388400 & 0.31535800 \\
$\mathrm{H}$ & 2.73950300 & -1.16816100 & 0.12070800 \\
$\mathrm{H}$ & 1.61379100 & -0.76048000 & 1.39980900 \\
$\mathrm{C}$ & 1.86391100 & 0.76224500 & -0.10976400 \\
$\mathrm{H}$ & 2.65773300 & 1.25003100 & 0.46857700 \\
$\mathrm{H}$ & 2.21023600 & 0.81531800 & -1.15343800 \\
\hline $\mathrm{E}_{\text {tot }}{ }^{\mathrm{a}}$ & \multicolumn{3}{|c}{-348.070240393} \\
$\mathrm{ZPE}^{\mathrm{b}}$ & \multicolumn{3}{|c}{0.155619} \\
$\mathrm{Imag}^{\mathrm{c}}$ & \multicolumn{3}{|c}{} \\
\hline $\mathrm{E}^{\mathrm{c}}$ & \multicolumn{3}{|c}{}
\end{tabular}

${ }^{\mathrm{a}} E_{\text {tot }}$ : electronic energy. ${ }^{\mathrm{b}} \mathrm{ZPE}$ : zero point energy, unscaled

${ }^{\mathrm{c}}$ Imag: imaginary frequency

\begin{tabular}{l|ccc} 
& \multicolumn{4}{|c}{ trans 18; B3LYP/6-311+G(d,p) } \\
\hline $\mathrm{C}$ & 0.40162300 & -1.09827100 & 0.64179300 \\
$\mathrm{C}$ & -0.49689300 & -1.35473200 & -0.32001600 \\
$\mathrm{H}$ & 0.02550100 & -0.88287400 & 1.64179700 \\
$\mathrm{H}$ & -0.14766600 & -1.44082900 & -1.34698100 \\
$\mathrm{C}$ & 1.47631800 & -0.17267600 & 0.17145200 \\
$\mathrm{O}$ & 2.52415400 & -0.40329300 & -0.37842000 \\
$\mathrm{C}$ & 0.86144100 & 1.24349600 & 0.30547600 \\
$\mathrm{H}$ & 0.74930100 & 1.48239100 & 1.37029700 \\
$\mathrm{H}$ & 1.57505300 & 1.95382700 & -0.11944400 \\
$\mathrm{C}$ & -0.52436900 & 1.40599300 & -0.40794600 \\
$\mathrm{H}$ & -0.75208800 & 2.47617500 & -0.37989600 \\
$\mathrm{H}$ & -0.39945000 & 1.16031200 & -1.46866100 \\
$\mathrm{C}$ & -1.78175600 & 0.66937600 & 0.16123500 \\
$\mathrm{H}$ & -1.80083000 & 0.82502800 & 1.24505200 \\
$\mathrm{H}$ & -2.66797200 & 1.17496000 & -0.23513300 \\
$\mathrm{C}$ & -1.91078700 & -0.87684700 & -0.11889300 \\
$\mathrm{H}$ & -2.52062600 & -1.06137600 & -1.00603300 \\
$\mathrm{H}$ & -2.40791500 & -1.35930000 & 0.72776500 \\
\hline $\mathrm{E}_{\text {tot }}{ }^{\mathrm{a}}$ & \multicolumn{3}{|c}{-348.014377628} \\
$\mathrm{ZPE}^{\mathrm{b}}$ & \multicolumn{3}{|c}{0.154500} \\
$\mathrm{Imag}^{\mathrm{c}}$ & \multicolumn{3}{|c}{} \\
\hline
\end{tabular}

${ }^{\mathrm{a}} E_{\text {tot }}$ : electronic energy. ${ }^{\mathrm{b}} \mathrm{ZPE}$ : zero point energy, unscaled

${ }^{\mathrm{c}}$ Imag: imaginary frequency 


\begin{tabular}{l|ccc} 
& \multicolumn{3}{|c}{ s-cis 1-methoxy-1,3-butadiene (14); B3LYP/6-311+G(d,p) } \\
\hline $\mathrm{C}$ & 0.00000000 & 0.00000000 & 0.00000000 \\
$\mathrm{H}$ & 1.09610630 & 0.00000000 & 0.00000000 \\
$\mathrm{H}$ & -0.35957216 & 1.02775833 & 0.00000000 \\
$\mathrm{H}$ & -0.36445460 & -0.51352596 & 0.89620613 \\
$\mathrm{O}$ & -0.51098484 & -0.60965633 & -1.18200217 \\
$\mathrm{C}$ & -0.05370781 & -1.86228115 & -1.43892403 \\
$\mathrm{H}$ & 0.86935569 & -2.14243223 & -0.93346073 \\
$\mathrm{C}$ & -0.68852652 & -2.69429763 & -2.27466610 \\
$\mathrm{H}$ & -1.64884747 & -2.38060732 & -2.67371418 \\
$\mathrm{C}$ & -0.18512126 & -4.01311221 & -2.65372137 \\
$\mathrm{H}$ & -0.94955469 & -4.76352813 & -2.84729079 \\
$\mathrm{C}$ & 1.09800513 & -4.37101935 & -2.79243597 \\
$\mathrm{H}$ & 1.90885358 & -3.65843896 & -2.68234481 \\
$\mathrm{H}$ & 1.37187748 & -5.39085077 & -3.03558646 \\
\hline $\mathrm{E}_{\text {tot }}{ }^{\mathrm{a}}$ & \multicolumn{3}{|c}{-270.592915992} \\
$\mathrm{ZPE}$ & \multicolumn{3}{|c}{0.117412} \\
$\mathrm{Imag}^{\mathrm{c}}$ & \multicolumn{3}{|c}{--}
\end{tabular}

${ }^{a} E_{\text {tot }}$ : electronic energy. ${ }^{b}$ ZPE: zero point energy, unscaled

${ }^{\mathrm{c}}$ Imag: imaginary frequency

\begin{tabular}{l|ccc} 
& s-cis 1-methoxy-1,3-butadiene (14); B3LYP/6-31G(d) \\
\hline $\mathrm{C}$ & 0.00000000 & 0.00000000 & 0.00000000 \\
$\mathrm{H}$ & 1.09896681 & 0.00000000 & 0.00000000 \\
$\mathrm{H}$ & -0.35842795 & 1.03118201 & 0.00000000 \\
$\mathrm{H}$ & -0.35884772 & -0.50896042 & 0.90443845 \\
$\mathrm{O}$ & -0.51574375 & -0.60895247 & -1.17662500 \\
$\mathrm{C}$ & -0.06174953 & -1.86522560 & -1.42796125 \\
$\mathrm{H}$ & 0.85795203 & -2.15035984 & -0.91453229 \\
$\mathrm{C}$ & -0.69519015 & -2.69569969 & -2.27146862 \\
$\mathrm{H}$ & -1.64936113 & -2.37280350 & -2.68329524 \\
$\mathrm{C}$ & -0.19684020 & -4.01965092 & -2.64312545 \\
$\mathrm{H}$ & -0.96639396 & -4.75450266 & -2.88359654 \\
$\mathrm{C}$ & 1.08706942 & -4.40209941 & -2.71824665 \\
$\mathrm{H}$ & 1.90659563 & -3.70654461 & -2.55405770 \\
$\mathrm{H}$ & 1.35806187 & -5.42635031 & -2.95691986 \\
\hline $\mathrm{E}_{\text {tot }}{ }^{\mathrm{a}}$ & \multicolumn{3}{|c}{-270.509819892} \\
$\mathrm{ZPE}$ & \multicolumn{3}{|c}{0.118556} \\
$\mathrm{Imag}^{\mathrm{c}}$ & \multicolumn{3}{|c}{--} \\
\hline
\end{tabular}

${ }^{\mathrm{a}} E_{\text {tot }}$ : electronic energy. ${ }^{\mathrm{b}} \mathrm{ZPE}$ : zero point energy, unscaled

${ }^{\mathrm{c}}$ Imag: imaginary frequency 


\begin{tabular}{|c|c|c|c|}
\hline & \multicolumn{3}{|c|}{ TS19a; B3LYP/6-311+G(d,p) } \\
\hline $\mathrm{C}$ & -0.52980300 & -2.47458300 & 0.16117300 \\
\hline $\mathrm{C}$ & -1.43506100 & -2.10101600 & 1.12257000 \\
\hline $\mathrm{C}$ & -2.33153100 & -1.00361200 & 1.03016600 \\
\hline $\mathrm{C}$ & -2.43776300 & -0.19117800 & -0.05964000 \\
\hline $\mathrm{C}$ & 0.18628500 & 0.50647600 & -0.18820600 \\
\hline $\mathrm{C}$ & 0.82472300 & -0.64849100 & 0.21386100 \\
\hline $\mathrm{H}$ & -2.89656300 & -0.71957900 & 1.91252200 \\
\hline $\mathrm{H}$ & -1.36598300 & -2.57832900 & 2.09634600 \\
\hline $\mathrm{H}$ & -0.65196300 & -2.23436400 & -0.88630300 \\
\hline $\mathrm{H}$ & -2.04184800 & -0.45561600 & -1.03234400 \\
\hline $\mathrm{H}$ & 0.12304900 & 0.69380000 & -1.26039800 \\
\hline $\mathrm{H}$ & 1.07170100 & -0.69362800 & 1.27247500 \\
\hline $\mathrm{C}$ & 1.97909200 & -0.93332400 & -0.68414600 \\
\hline $\mathrm{O}$ & 1.99001900 & -1.61403400 & -1.68653300 \\
\hline C & 3.18313700 & -0.05206900 & -0.28172600 \\
\hline $\mathrm{H}$ & 3.56225200 & 0.36124100 & -1.22282500 \\
\hline $\mathrm{H}$ & 3.97192000 & -0.71804600 & 0.08917200 \\
\hline C & 2.95652900 & 1.08756200 & 0.75276800 \\
\hline $\mathrm{H}$ & 3.91273600 & 1.61519300 & 0.83603500 \\
\hline $\mathrm{H}$ & 2.78041100 & 0.65553200 & 1.74413300 \\
\hline C & 1.87010800 & 2.16375000 & 0.47121400 \\
\hline $\mathrm{H}$ & 1.99130400 & 2.53252800 & -0.55487000 \\
\hline $\mathrm{H}$ & 2.06951700 & 3.01883000 & 1.12772300 \\
\hline C & 0.38673500 & 1.72354200 & 0.67093300 \\
\hline $\mathrm{H}$ & 0.21608500 & 1.48114800 & 1.72579900 \\
\hline $\mathrm{H}$ & -0.27892000 & 2.55261800 & 0.41117900 \\
\hline $\mathrm{O}$ & -3.23490300 & 0.90373900 & -0.02097200 \\
\hline $\mathrm{H}$ & 0.15615500 & -3.29068300 & 0.35621600 \\
\hline C & -3.38136600 & 1.60313700 & -1.25405500 \\
\hline $\mathrm{H}$ & -4.04469000 & 2.44354500 & -1.05668000 \\
\hline $\mathrm{H}$ & -3.82800000 & 0.96000000 & -2.01981200 \\
\hline $\mathrm{H}$ & -2.41460300 & 1.97700800 & -1.60780200 \\
\hline$E_{\text {tot }}^{a}$ & & 618.599695417 & \\
\hline $\mathrm{ZPE}^{\mathrm{b}}$ & & 0.273689 & \\
\hline $\operatorname{Imag}^{c}$ & & -281.1579 & \\
\hline
\end{tabular}




\begin{tabular}{|c|c|c|c|}
\hline & \multicolumn{3}{|c|}{ TS19b; B3LYP/6-311+G(d,p) } \\
\hline $\mathrm{C}$ & 2.30286100 & -0.44766700 & 0.53497900 \\
\hline $\mathrm{C}$ & 2.17260800 & -1.16607000 & -0.62439000 \\
\hline $\mathrm{C}$ & 1.27247300 & -2.25412300 & -0.76580600 \\
\hline $\mathrm{C}$ & 0.33869100 & -2.65380400 & 0.16264600 \\
\hline $\mathrm{C}$ & -1.04375100 & -0.89088600 & 0.04760600 \\
\hline $\mathrm{C}$ & -0.42083100 & 0.18154400 & 0.66123100 \\
\hline $\mathrm{H}$ & 1.21489800 & -2.69801200 & -1.75615700 \\
\hline $\mathrm{H}$ & 2.71201300 & -0.84714200 & -1.50819600 \\
\hline $\mathrm{H}$ & 1.90223800 & -0.80063500 & 1.47463300 \\
\hline $\mathrm{H}$ & 0.45587700 & -2.44718500 & 1.21823000 \\
\hline $\mathrm{H}$ & -0.31298300 & -3.48781300 & -0.07056900 \\
\hline $\mathrm{H}$ & -1.04406400 & -0.85131000 & -1.04077400 \\
\hline $\mathrm{H}$ & -0.33719000 & 0.21201000 & 1.74685400 \\
\hline $\mathrm{C}$ & -0.52824400 & 1.51048900 & 0.03485100 \\
\hline $\mathrm{O}$ & 0.13042900 & 2.49293000 & 0.34125200 \\
\hline $\mathrm{C}$ & -1.79340000 & 1.63830500 & -0.83750300 \\
\hline $\mathrm{H}$ & -1.65954600 & 1.10812300 & -1.78846400 \\
\hline $\mathrm{H}$ & -1.91583000 & 2.69775300 & -1.07303000 \\
\hline $\mathrm{C}$ & -3.07167400 & 1.10889900 & -0.10635200 \\
\hline $\mathrm{H}$ & -3.93408800 & 1.60935900 & -0.55956200 \\
\hline $\mathrm{H}$ & -3.04048900 & 1.45532500 & 0.93335000 \\
\hline $\mathrm{C}$ & -3.38764500 & -0.41133900 & -0.13345600 \\
\hline $\mathrm{H}$ & -3.48303600 & -0.72363300 & 1.18058500 \\
\hline $\mathrm{H}$ & -4.37422600 & -0.55897100 & 0.32085300 \\
\hline $\mathrm{C}$ & -2.37234600 & -1.37834300 & 0.55779600 \\
\hline $\mathrm{H}$ & -2.43315400 & -1.29201500 & 1.64687700 \\
\hline $\mathrm{H}$ & -2.61922800 & -2.40804800 & 0.28016100 \\
\hline $\mathrm{O}$ & 3.04996400 & 0.64158000 & 0.73304300 \\
\hline $\mathrm{C}$ & 3.57808400 & 1.33020700 & -0.40219900 \\
\hline $\mathrm{H}$ & 4.04454200 & 2.23090200 & -0.00961600 \\
\hline $\mathrm{H}$ & 2.77302900 & 1.60239400 & -1.08881800 \\
\hline $\mathrm{H}$ & 4.32714800 & 0.71955500 & -0.91596600 \\
\hline $\mathrm{E}_{\text {tot }}^{\mathrm{a}}$ & & 618.599110700 & \\
\hline $\mathrm{ZPE}^{\mathrm{b}}$ & & 0.274561 & \\
\hline $\operatorname{Imag}^{c}$ & & -314.0790 & \\
\hline
\end{tabular}




\begin{tabular}{|c|c|c|c|}
\hline & \multicolumn{3}{|c|}{ TS19c; B3LYP/6-311+G(d,p) } \\
\hline $\mathrm{C}$ & 2.10266200 & 0.10182400 & -0.38103900 \\
\hline $\mathrm{C}$ & 2.32617700 & -1.02629900 & 0.38199800 \\
\hline $\mathrm{C}$ & 1.69850400 & -2.26855000 & 0.13390100 \\
\hline $\mathrm{C}$ & 0.83771900 & -2.52139600 & -0.90515700 \\
\hline $\mathrm{C}$ & -0.88229400 & -0.99771100 & -0.46599500 \\
\hline $\mathrm{C}$ & -0.28808300 & 0.15790700 & 0.01909900 \\
\hline $\mathrm{H}$ & 1.79382800 & -3.01976000 & 0.91348500 \\
\hline $\mathrm{H}$ & 2.89060200 & -0.93144700 & 1.30188400 \\
\hline $\mathrm{H}$ & 1.72225800 & 0.06689800 & -1.39139300 \\
\hline $\mathrm{H}$ & 0.89145200 & -1.96046000 & -1.82821400 \\
\hline $\mathrm{H}$ & 0.34226700 & -3.48295600 & -0.97555100 \\
\hline $\mathrm{H}$ & -1.17596000 & -0.98444300 & -1.51485600 \\
\hline $\mathrm{H}$ & -0.24199200 & 0.23590500 & 1.10355000 \\
\hline C & -0.86331700 & 1.34354000 & -0.66378400 \\
\hline $\mathrm{O}$ & -0.51158500 & 1.82613100 & -1.71944100 \\
\hline $\mathrm{C}$ & -2.16898700 & 1.77918200 & 0.03818800 \\
\hline $\mathrm{H}$ & -2.85903900 & 2.04514900 & -0.76920600 \\
\hline $\mathrm{H}$ & -1.96810500 & 2.71083400 & 0.58165900 \\
\hline C & -2.83552200 & 0.76270100 & 1.01125300 \\
\hline $\mathrm{H}$ & -3.80240600 & 1.19569100 & 1.28948300 \\
\hline $\mathrm{H}$ & -2.26218400 & 0.71584600 & 1.94363000 \\
\hline C & -3.11237200 & -0.68692500 & 0.52011800 \\
\hline $\mathrm{H}$ & -3.58764500 & -0.64551000 & -0.46773700 \\
\hline $\mathrm{H}$ & -3.85443700 & -1.12940100 & 1.19437400 \\
\hline C & -1.89053800 & -1.65314100 & 0.44383000 \\
\hline $\mathrm{H}$ & -1.46976900 & -1.79411000 & 1.44545500 \\
\hline $\mathrm{H}$ & -2.22258500 & -2.63029700 & 0.07956500 \\
\hline $\mathrm{O}$ & 2.61116800 & 1.32874000 & -0.13253300 \\
\hline C & 3.22095900 & 1.57498400 & 1.12927100 \\
\hline $\mathrm{H}$ & 3.46858400 & 2.63501100 & 1.13811900 \\
\hline $\mathrm{H}$ & 4.13647200 & 0.98660800 & 1.25033600 \\
\hline $\mathrm{H}$ & 2.53253700 & 1.35079000 & 1.95110100 \\
\hline$E_{\text {tot }}^{a}$ & & 618.597486880 & \\
\hline $\mathrm{ZPE}^{\mathrm{b}}$ & & 0.274521 & \\
\hline $\operatorname{Imag}^{c}$ & & -323.5691 & \\
\hline
\end{tabular}




\begin{tabular}{|c|c|c|c|}
\hline & \multicolumn{3}{|c|}{ TS19d; B3LYP/6-311+G(d,p) } \\
\hline $\mathrm{C}$ & 0.51016300 & -2.13176600 & -1.18583500 \\
\hline $\mathrm{C}$ & 1.27482000 & -2.34734000 & -0.06592000 \\
\hline $\mathrm{C}$ & 2.22327000 & -1.43315900 & 0.45969900 \\
\hline $\mathrm{C}$ & 2.46217200 & -0.19450700 & -0.05857500 \\
\hline $\mathrm{C}$ & -0.18397300 & 0.52764400 & 0.19028100 \\
\hline $\mathrm{C}$ & -0.82352100 & -0.43616700 & -0.56640600 \\
\hline $\mathrm{H}$ & 2.71079700 & -1.67369600 & 1.39928100 \\
\hline $\mathrm{H}$ & 1.03045700 & -3.20133100 & 0.55703600 \\
\hline $\mathrm{H}$ & 0.84863900 & -1.47552600 & -1.97837300 \\
\hline $\mathrm{H}$ & 2.11391000 & 0.11918400 & -1.03514000 \\
\hline $\mathrm{H}$ & -0.03797600 & 0.32626700 & 1.24928500 \\
\hline $\mathrm{H}$ & -1.12921300 & -0.10195600 & -1.55900600 \\
\hline $\mathrm{C}$ & -1.94539700 & -1.06610100 & 0.19847200 \\
\hline $\mathrm{O}$ & -1.92796500 & -2.08144800 & 0.85535300 \\
\hline $\mathrm{C}$ & -3.17190200 & -0.12348600 & 0.20422500 \\
\hline $\mathrm{H}$ & -3.49794300 & -0.08535900 & 1.24947500 \\
\hline $\mathrm{H}$ & -3.97682000 & -0.63455800 & -0.33801900 \\
\hline C & -3.01703000 & 1.31676400 & -0.35905700 \\
\hline $\mathrm{H}$ & -3.98549700 & 1.80653200 & -0.21078300 \\
\hline $\mathrm{H}$ & -2.87943800 & 1.27595900 & -1.44554700 \\
\hline C & -1.93801300 & 2.25127800 & 0.25167800 \\
\hline $\mathrm{H}$ & -2.00568500 & 2.21027900 & 1.34548700 \\
\hline $\mathrm{H}$ & -2.18518300 & 3.28140600 & -0.03165500 \\
\hline C & -0.46373700 & 1.96278800 & -0.16567100 \\
\hline $\mathrm{H}$ & -0.36052100 & 2.12742800 & -1.24560300 \\
\hline $\mathrm{H}$ & 0.19782500 & 2.66940300 & 0.34581200 \\
\hline $\mathrm{O}$ & 3.30840700 & 0.65604100 & 0.57124300 \\
\hline $\mathrm{H}$ & -0.22848000 & -2.86871400 & -1.47639200 \\
\hline C & 3.61282400 & 1.85935600 & -0.12886300 \\
\hline $\mathrm{H}$ & 4.27184100 & 2.43559600 & 0.51848600 \\
\hline $\mathrm{H}$ & 2.70396900 & 2.43569200 & -0.32957000 \\
\hline $\mathrm{H}$ & 4.12771800 & 1.64483300 & -1.07170500 \\
\hline$E_{\text {tot }}^{a}$ & & 518.597544075 & \\
\hline $\mathrm{ZPE}^{\mathrm{b}}$ & & 0.273761 & \\
\hline $\operatorname{Imag}^{c}$ & & -297.0127 & \\
\hline
\end{tabular}




\begin{tabular}{|c|c|c|c|}
\hline & \multicolumn{3}{|c|}{ TS20a; B3LYP/6-311+G(d,p) } \\
\hline $\mathrm{C}$ & -1.00102000 & 1.06153600 & 0.94373500 \\
\hline $\mathrm{H}$ & -0.84778800 & 1.71380800 & 1.79969300 \\
\hline $\mathrm{C}$ & -0.19709000 & -0.10316700 & 1.03148600 \\
\hline $\mathrm{H}$ & 0.58688800 & -0.08513600 & 1.77982800 \\
\hline $\mathrm{H}$ & -0.85420600 & 3.22857700 & -0.23105800 \\
\hline $\mathrm{C}$ & -0.22666400 & 2.35180600 & -0.35815300 \\
\hline $\mathrm{H}$ & -0.50375000 & 1.76227700 & -1.22516700 \\
\hline $\mathrm{C}$ & 1.13535300 & 2.50066400 & -0.05375500 \\
\hline $\mathrm{H}$ & 1.44561600 & 3.38729400 & 0.49122400 \\
\hline $\mathrm{C}$ & 2.06871500 & 1.46749200 & -0.17284900 \\
\hline $\mathrm{H}$ & 3.04258200 & 1.59368300 & 0.28576800 \\
\hline $\mathrm{C}$ & 1.73298400 & 0.24456600 & -0.72863000 \\
\hline $\mathrm{H}$ & 0.88543700 & 0.14250000 & -1.39036800 \\
\hline $\mathrm{O}$ & 2.48892100 & -0.85336300 & -0.77424300 \\
\hline $\mathrm{C}$ & -0.38062000 & -1.44843400 & 0.50470500 \\
\hline $\mathrm{O}$ & 0.29664800 & -2.37136800 & 0.96286700 \\
\hline $\mathrm{C}$ & -1.33372400 & -1.75909300 & -0.65019600 \\
\hline $\mathrm{H}$ & -0.88452700 & -1.36442200 & -1.57288900 \\
\hline $\mathrm{H}$ & -1.32743400 & -2.84650700 & -0.74633700 \\
\hline $\mathrm{C}$ & -2.77580800 & -1.24734200 & -0.54632200 \\
\hline $\mathrm{H}$ & -3.35265800 & -1.72182700 & -1.34740400 \\
\hline $\mathrm{H}$ & -3.22342500 & -1.59068900 & 0.39426700 \\
\hline $\mathrm{C}$ & -2.93465200 & 0.27072700 & -0.66669400 \\
\hline $\mathrm{H}$ & -3.99309000 & 0.50105200 & -0.83160100 \\
\hline $\mathrm{H}$ & -2.41200600 & 0.62089400 & -1.56596400 \\
\hline C & -2.48115600 & 1.06649500 & 0.56072200 \\
\hline $\mathrm{H}$ & -2.79958500 & 2.10724700 & 0.44138000 \\
\hline $\mathrm{H}$ & -3.03016000 & 0.68980400 & 1.43422900 \\
\hline C & 3.61183600 & -0.95907900 & 0.10562300 \\
\hline $\mathrm{H}$ & 3.30453000 & -0.77619500 & 1.13787500 \\
\hline $\mathrm{H}$ & 3.96846900 & -1.98141200 & 0.00517700 \\
\hline $\mathrm{H}$ & 4.40163400 & -0.26013100 & -0.18567600 \\
\hline $\mathrm{E}_{\text {tot }}^{\mathrm{a}}$ & & 518.626883385 & \\
\hline $\mathrm{ZPE}^{\mathrm{b}}$ & & 0.276272 & \\
\hline $\operatorname{Imag}^{c}$ & & -475.1483 & \\
\hline
\end{tabular}




\begin{tabular}{l|ccc} 
& \multicolumn{3}{|c}{ TS20b; B3LYP/6-311+G(d,p) } \\
\hline $\mathrm{C}$ & -0.08799200 & -0.08109300 & 1.08772200 \\
$\mathrm{H}$ & -0.66564500 & 0.21566200 & 1.95707400 \\
$\mathrm{C}$ & 0.94345800 & 0.82936800 & 0.76574400 \\
$\mathrm{H}$ & 1.02869700 & 1.68984300 & 1.41725700 \\
$\mathrm{C}$ & -1.88446400 & 0.37143900 & -0.14663200 \\
$\mathrm{H}$ & -1.35856200 & -0.08627800 & -0.97932500 \\
$\mathrm{C}$ & -1.97428400 & 1.75633800 & -0.02370500 \\
$\mathrm{H}$ & -2.81699700 & 2.14403800 & 0.54004300 \\
$\mathrm{C}$ & -0.92679700 & 2.60575200 & -0.37378000 \\
$\mathrm{H}$ & -0.97508300 & 3.63880400 & -0.04344300 \\
$\mathrm{C}$ & 0.26762200 & 2.12396400 & -0.89496400 \\
$\mathrm{H}$ & 0.26347600 & 1.25993900 & -1.54562200 \\
$\mathrm{C}$ & 2.27520300 & 0.50217300 & 0.18896300 \\
$\mathrm{O}$ & 3.25252400 & 1.13450600 & 0.56139800 \\
$\mathrm{C}$ & 2.44306800 & -0.56284700 & -0.88944700 \\
$\mathrm{H}$ & 1.85038800 & -0.27188900 & -1.76617200 \\
$\mathrm{H}$ & 3.49372900 & -0.52340100 & -1.18288800 \\
$\mathrm{C}$ & 2.06244200 & -1.99647300 & -0.48934200 \\
$\mathrm{H}$ & 2.42345000 & -2.66487600 & -1.27783500 \\
$\mathrm{H}$ & 2.60222200 & -2.28061700 & 0.42182400 \\
$\mathrm{C}$ & 0.56220100 & -2.23086200 & -0.29480500 \\
$\mathrm{H}$ & 0.38418400 & -3.31015800 & -0.23240000 \\
$\mathrm{H}$ & 0.02704100 & -1.89179800 & -1.19150500 \\
$\mathrm{C}$ & -0.04156700 & -1.59348600 & 0.96217700 \\
$\mathrm{H}$ & -1.06289500 & -1.96287600 & 1.09586900 \\
$\mathrm{H}$ & 0.51156200 & -1.97250400 & 1.83424300 \\
$\mathrm{O}$ & -2.88477500 & -0.39678500 & 0.39462800 \\
$\mathrm{H}$ & 1.09977500 & 2.80150600 & -1.04932900 \\
$\mathrm{C}$ & -3.45675900 & -1.36340900 & -0.48391200 \\
$\mathrm{H}$ & -4.14133300 & -1.96368200 & 0.11432000 \\
$\mathrm{H}$ & -4.01136300 & -0.87467800 & -1.29261900 \\
$\mathrm{H}$ & -2.68741700 & -2.01398300 & -0.91582100 \\
\hline $\mathrm{E}_{\text {tot }}{ }^{\mathrm{b}}$ & & -618.619641747 & \\
$\mathrm{ZPE}$ & & 0.275916 & \\
$\mathrm{Imag}$ & & -537.6403 & \\
\hline $\mathrm{a} \mathrm{E}_{\text {tot }}:$ electronic energy. & \\
${ }^{\mathrm{I}} \mathrm{Imag}: \mathrm{ZPE}:$ zero point & imaginary frequency & & \\
& & & \\
& & & \\
$\mathrm{H}$ & & & \\
$\mathrm{H}$ & &
\end{tabular}




\begin{tabular}{l|ccc} 
& \multicolumn{3}{|c}{ cis 9 ; B BLYP/6-31G(d) } \\
\hline $\mathrm{C}$ & 0.00000000 & 0.00000000 & 0.00000000 \\
$\mathrm{H}$ & 1.09157778 & 0.00000000 & 0.00000000 \\
$\mathrm{C}$ & -0.56862870 & 1.21739339 & 0.00000000 \\
$\mathrm{H}$ & 0.09186125 & 2.08250801 & -0.00338471 \\
$\mathrm{C}$ & -1.98366267 & 1.65704238 & 0.07991606 \\
$\mathrm{O}$ & -2.17615762 & 2.85728390 & 0.23823151 \\
$\mathrm{C}$ & -3.20854257 & 0.74489503 & -0.14245654 \\
$\mathrm{H}$ & -3.40935967 & 0.88007035 & -1.21899564 \\
$\mathrm{C}$ & -3.13482349 & -0.78104164 & 0.09998997 \\
$\mathrm{C}$ & -1.97577363 & -1.49924392 & -0.60874156 \\
$\mathrm{H}$ & -2.22657385 & -2.56825175 & -0.65928362 \\
$\mathrm{H}$ & -1.90119306 & -1.14995693 & -1.64773532 \\
$\mathrm{C}$ & -0.59865631 & -1.38469602 & 0.07467603 \\
$\mathrm{C}$ & -4.42109611 & 1.30205191 & 0.61888528 \\
$\mathrm{H}$ & -4.41863017 & 2.39141031 & 0.57875858 \\
$\mathrm{H}$ & -5.34883997 & 0.93530465 & 0.15500320 \\
$\mathrm{O}$ & -4.37137287 & 0.96549746 & 1.99764823 \\
$\mathrm{C}$ & -4.40786688 & -0.44116306 & 2.30191887 \\
$\mathrm{C}$ & -5.75583092 & -1.06238347 & 1.87777984 \\
$\mathrm{H}$ & -5.85809989 & -2.06634942 & 2.30451687 \\
$\mathrm{H}$ & -6.57785859 & -0.44447123 & 2.25388484 \\
$\mathrm{H}$ & -5.87142000 & -1.15573677 & 0.79412267 \\
$\mathrm{C}$ & -4.32143373 & -0.48536096 & 3.83183909 \\
$\mathrm{H}$ & -5.22513289 & -0.04153005 & 4.26177427 \\
$\mathrm{H}$ & -4.23659463 & -1.51589493 & 4.19485777 \\
$\mathrm{H}$ & -3.46464572 & 0.08903010 & 4.19242088 \\
$\mathrm{H}$ & -4.04720022 & -1.16558330 & -0.37630797 \\
$\mathrm{C}$ & -3.21692648 & -1.17041739 & 1.60288447 \\
$\mathrm{H}$ & -3.44521152 & -2.24787928 & 1.63482984 \\
$\mathrm{C}$ & -1.85469124 & -0.98887820 & 2.29823471 \\
$\mathrm{H}$ & -1.91003993 & -1.35072467 & 3.33035814 \\
$\mathrm{H}$ & -1.59526142 & 0.07486472 & 2.35930800 \\
$\mathrm{C}$ & -0.74852531 & -1.76747917 & 1.57564124 \\
$\mathrm{H}$ & -0.97443188 & -2.84346234 & 1.62430911 \\
$\mathrm{H}$ & 0.21139311 & -1.62871343 & 2.09021213 \\
$\mathrm{C}$ & 0.37915217 & -2.36598605 & -0.60576509 \\
$\mathrm{H}$ & 0.51802713 & -2.11764119 & -1.66489156 \\
$\mathrm{H}$ & 1.36363957 & -2.34259795 & -0.12295620 \\
$\mathrm{H}$ & 0.00079336 & -3.39286080 & -0.54674792 \\
\hline $\mathrm{E}_{\text {tot }}{ }^{\mathrm{a}}$ & & -735.273892791 & \\
$\mathrm{ZPE} \mathrm{b}$ & & 0.348154 & \\
$\mathrm{Imag}$ & & -- & \\
\hline $\mathrm{a}$ & & & \\
\hline & & &
\end{tabular}

\footnotetext{
${ }^{a} E_{\text {tot }}$ : electronic energy. ${ }^{b} \mathrm{ZPE}$ : zero point energy, unscaled

${ }^{\mathrm{c}}$ Imag: imaginary frequency
} 


\begin{tabular}{l|crc} 
& \multicolumn{3}{|c}{ trans $9 \mathrm{a} ; \mathrm{B} 3 \mathrm{LYP} / 6-31 \mathrm{G}(\mathrm{d})$} \\
\hline $\mathrm{C}$ & 0.00000000 & 0.00000000 & 0.00000000 \\
$\mathrm{O}$ & 1.21092696 & 0.00000000 & 0.00000000 \\
$\mathrm{C}$ & -0.80561961 & 1.34585665 & 0.00000000 \\
$\mathrm{H}$ & -0.18401418 & 1.97631090 & -0.65139409 \\
$\mathrm{C}$ & -2.28621158 & 1.42747074 & -0.56740607 \\
$\mathrm{C}$ & -2.63153838 & 0.70586788 & -1.90437296 \\
$\mathrm{H}$ & -3.56922983 & 1.14693341 & -2.27605073 \\
$\mathrm{H}$ & -1.86805105 & 0.92755585 & -2.66178207 \\
$\mathrm{C}$ & -2.83956532 & -0.84826433 & -1.80135771 \\
$\mathrm{C}$ & -0.77394980 & 1.99110189 & 1.40042196 \\
$\mathrm{H}$ & 0.16710626 & 1.75068773 & 1.90384332 \\
$\mathrm{H}$ & -0.82602967 & 3.08518636 & 1.30683156 \\
$\mathrm{O}$ & -1.80797705 & 1.50861520 & 2.24342506 \\
$\mathrm{C}$ & -3.14961308 & 1.84471966 & 1.82649641 \\
$\mathrm{C}$ & -3.34384610 & 3.37342708 & 1.73761273 \\
$\mathrm{H}$ & -4.41192005 & 3.60544364 & 1.66636389 \\
$\mathrm{H}$ & -2.95125872 & 3.84980239 & 2.64233004 \\
$\mathrm{H}$ & -2.85471075 & 3.83007929 & 0.87245178 \\
$\mathrm{C}$ & -4.02848149 & 1.32652786 & 2.97084424 \\
$\mathrm{H}$ & -3.81448861 & 1.89779590 & 3.87982751 \\
$\mathrm{H}$ & -5.09206316 & 1.43999732 & 2.73463121 \\
$\mathrm{H}$ & -3.82583345 & 0.27445114 & 3.18423024 \\
$\mathrm{H}$ & -2.36712342 & 2.49458779 & -0.81522335 \\
$\mathrm{C}$ & -3.44776486 & 1.16773358 & 0.44945186 \\
$\mathrm{H}$ & -4.30745802 & 1.70360328 & 0.01845467 \\
$\mathrm{C}$ & -3.90049705 & -0.30897300 & 0.57156085 \\
$\mathrm{H}$ & -4.91435159 & -0.31731661 & 0.98975721 \\
$\mathrm{H}$ & -3.29351689 & -0.83072683 & 1.31456982 \\
$\mathrm{C}$ & -3.95195528 & -1.10590706 & -0.75697439 \\
$\mathrm{H}$ & -4.89781262 & -0.85961629 & -1.25887302 \\
$\mathrm{H}$ & -4.00454008 & -2.17884774 & -0.53103185 \\
$\mathrm{C}$ & -3.25271127 & -1.42714050 & -3.16214233 \\
$\mathrm{H}$ & -2.47281016 & -1.26136920 & -3.91525706 \\
$\mathrm{H}$ & -3.42268287 & -2.50791948 & -3.09033059 \\
$\mathrm{H}$ & -4.17651747 & -0.96328290 & -3.52836986 \\
$\mathrm{H}$ & -0.70772911 & -1.06245930 & -2.21758069 \\
$\mathrm{C}$ & -1.42851236 & -1.23430505 & -1.41266155 \\
$\mathrm{C}$ & -0.98684760 & -1.10011504 & -0.15201843 \\
$\mathrm{H}$ & -1.69025001 & -1.15007758 & 0.66695212 \\
\hline $\mathrm{E}_{\text {tot }}{ }^{\mathrm{a}}$ & & -735.204396549 & \\
$\mathrm{ZPE} \mathrm{b}$ & & 0.347058 & \\
$\mathrm{Imag}$ & & -- & \\
\hline $\mathrm{a}$ & & & \\
\hline & & &
\end{tabular}

\footnotetext{
${ }^{a} E_{\text {tot }}$ : electronic energy. ${ }^{b} \mathrm{ZPE}$ : zero point energy, unscaled

${ }^{\mathrm{c}}$ Imag: imaginary frequency
} 


\begin{tabular}{l|ccc} 
& \multicolumn{3}{|c}{ trans 9 b; B3LYP/6-31G(d) } \\
\hline $\mathrm{C}$ & 0.00000000 & 0.00000000 & 0.00000000 \\
$\mathrm{O}$ & 1.20968609 & 0.00000000 & 0.00000000 \\
$\mathrm{C}$ & -0.88494639 & 1.29851007 & 0.00000000 \\
$\mathrm{H}$ & -1.41492609 & 1.27307818 & -0.96595573 \\
$\mathrm{C}$ & -2.02011819 & 1.44777423 & 1.10908782 \\
$\mathrm{C}$ & -3.00371398 & 0.26670302 & 1.39785248 \\
$\mathrm{H}$ & -3.83484785 & 0.69237052 & 1.97910353 \\
$\mathrm{H}$ & -3.45378533 & -0.09997135 & 0.46799527 \\
$\mathrm{C}$ & -2.40620708 & -0.91389672 & 2.22926239 \\
$\mathrm{C}$ & 0.00177080 & 2.55149523 & 0.01557300 \\
$\mathrm{H}$ & 0.90159786 & 2.37300852 & -0.57693984 \\
$\mathrm{H}$ & -0.54359451 & 3.39660528 & -0.43066584 \\
$\mathrm{O}$ & 0.46709929 & 2.87602124 & 1.31416016 \\
$\mathrm{C}$ & -0.54735038 & 3.21271838 & 2.27681912 \\
$\mathrm{C}$ & -1.33266691 & 4.46982286 & 1.84376082 \\
$\mathrm{H}$ & -1.94010806 & 4.83718869 & 2.67827974 \\
$\mathrm{H}$ & -0.63103642 & 5.26056264 & 1.55783184 \\
$\mathrm{H}$ & -2.00846948 & 4.29640984 & 1.00090359 \\
$\mathrm{C}$ & 0.25044174 & 3.56161151 & 3.53853484 \\
$\mathrm{H}$ & 0.83632902 & 4.46896366 & 3.35992869 \\
$\mathrm{H}$ & -0.41427417 & 3.74193871 & 4.39084117 \\
$\mathrm{H}$ & 0.94744880 & 2.76034201 & 3.79540988 \\
$\mathrm{H}$ & -2.65739091 & 2.23392162 & 0.68300760 \\
$\mathrm{C}$ & -1.51077263 & 2.00088290 & 2.47595342 \\
$\mathrm{H}$ & -2.40596271 & 2.39614963 & 2.98226070 \\
$\mathrm{C}$ & -0.95391155 & 0.88798401 & 3.38877211 \\
$\mathrm{H}$ & -0.74352426 & 1.30294592 & 4.38024098 \\
$\mathrm{H}$ & 0.00984556 & 0.54067215 & 2.99813164 \\
$\mathrm{C}$ & -1.93034083 & -0.29496500 & 3.57035045 \\
$\mathrm{H}$ & -2.81659012 & 0.04918730 & 4.12310684 \\
$\mathrm{H}$ & -1.45733421 & -1.07021821 & 4.18856026 \\
$\mathrm{C}$ & -3.44165886 & -2.02261788 & 2.46094080 \\
$\mathrm{H}$ & -3.76905057 & -2.45998058 & 1.51051350 \\
$\mathrm{H}$ & -3.02157914 & -2.82923612 & 3.07306712 \\
$\mathrm{H}$ & -4.32805540 & -1.63308585 & 2.97734948 \\
$\mathrm{H}$ & -1.88191195 & -0.98603381 & -0.40569025 \\
$\mathrm{C}$ & -0.98302833 & -1.10228317 & 0.19540247 \\
$\mathrm{C}$ & -1.13240077 & -1.35340662 & 1.50987721 \\
$\mathrm{H}$ & -0.22888031 & -1.40695233 & 2.11660693 \\
\hline $\mathrm{E}_{\text {tot }}{ }^{\mathrm{a}}$ & & -735.207828948 & \\
$\mathrm{ZPE} \mathrm{b}$ & & 0.347296 & \\
$\mathrm{Imag}$ & & -- & \\
\hline $\mathrm{a}$ & & & \\
\hline & & &
\end{tabular}

\footnotetext{
${ }^{\mathrm{a}} E_{\text {tot }}$ : electronic energy. ${ }^{\mathrm{b}} \mathrm{ZPE}$ : zero point energy, unscaled

${ }^{\mathrm{c}}$ Imag: imaginary frequency
} 
TS16a; B3LYP/6-31G(d)

\begin{tabular}{|c|c|c|c|}
\hline & \multicolumn{3}{|c|}{ TS16a; B3LYP/6-31G(d) } \\
\hline $\mathrm{C}$ & 0.00000000 & 0.00000000 & 0.00000000 \\
\hline $\mathrm{O}$ & 1.21795860 & 0.00000000 & 0.00000000 \\
\hline $\mathrm{C}$ & -0.79066414 & 1.33911184 & 0.00000000 \\
\hline $\mathrm{H}$ & -0.10060941 & 2.01271438 & -0.52544654 \\
\hline $\mathrm{C}$ & -2.18324583 & 1.44718119 & -0.74004595 \\
\hline $\mathrm{C}$ & -2.32296035 & 0.74871712 & -2.11895138 \\
\hline $\mathrm{H}$ & -3.18933608 & 1.20173705 & -2.62599847 \\
\hline $\mathrm{H}$ & -1.44844588 & 0.97643284 & -2.74354595 \\
\hline $\mathrm{C}$ & -2.54297482 & -0.80017568 & -2.07123470 \\
\hline $\mathrm{C}$ & -0.92756139 & 1.89128314 & 1.43664058 \\
\hline $\mathrm{H}$ & -0.06688886 & 1.59075024 & 2.04198384 \\
\hline $\mathrm{H}$ & -0.93701368 & 2.99025959 & 1.40843054 \\
\hline $\mathrm{O}$ & -2.07300726 & 1.39893272 & 2.11244046 \\
\hline $\mathrm{C}$ & -3.33939043 & 1.78843485 & 1.53640748 \\
\hline $\mathrm{C}$ & -3.48400101 & 3.32431707 & 1.48054528 \\
\hline $\mathrm{H}$ & -4.52626487 & 3.58845947 & 1.27136627 \\
\hline $\mathrm{H}$ & -3.21081597 & 3.75828238 & 2.44847841 \\
\hline $\mathrm{H}$ & -2.86896054 & 3.79718396 & 0.70986817 \\
\hline $\mathrm{C}$ & -4.37437759 & 1.25822724 & 2.53596639 \\
\hline $\mathrm{H}$ & -4.26777791 & 1.79152578 & 3.48616840 \\
\hline $\mathrm{H}$ & -5.39436443 & 1.41165111 & 2.16691974 \\
\hline $\mathrm{H}$ & -4.22868038 & 0.19382182 & 2.73355085 \\
\hline $\mathrm{H}$ & -2.23482359 & 2.51958415 & -0.97195169 \\
\hline $\mathrm{C}$ & -3.46677780 & 1.16287490 & 0.11150654 \\
\hline $\mathrm{H}$ & -4.26376373 & 1.71951197 & -0.40544180 \\
\hline $\mathrm{C}$ & -3.93357963 & -0.31357892 & 0.13135148 \\
\hline $\mathrm{H}$ & -4.99454782 & -0.32812652 & 0.40977665 \\
\hline $\mathrm{H}$ & -3.43130302 & -0.85810398 & 0.93361296 \\
\hline C & -3.80191295 & -1.07148792 & -1.21181949 \\
\hline $\mathrm{H}$ & -4.66304355 & -0.79561019 & -1.83670859 \\
\hline $\mathrm{H}$ & -3.89383093 & -2.14956191 & -1.02921327 \\
\hline C & -2.77937744 & -1.33413863 & -3.49361313 \\
\hline $\mathrm{H}$ & -1.90416294 & -1.15303819 & -4.13088983 \\
\hline $\mathrm{H}$ & -2.96693323 & -2.41316061 & -3.47297568 \\
\hline $\mathrm{H}$ & -3.63989307 & -0.84488487 & -3.96649795 \\
\hline $\mathrm{H}$ & -0.39125582 & -1.13407454 & -2.20789951 \\
\hline C & -1.22006281 & -1.29866767 & -1.51201640 \\
\hline $\mathrm{C}$ & -0.92093328 & -1.15074425 & -0.17337067 \\
\hline $\mathrm{H}$ & -1.75079342 & -1.12144817 & 0.51556775 \\
\hline C & -0.78681116 & -3.86653920 & 0.67663447 \\
\hline $\mathrm{H}$ & -1.31027547 & -4.11235192 & 1.59937766 \\
\hline C & 0.18207535 & -2.89516877 & 0.72735611 \\
\hline $\mathrm{H}$ & 0.48294225 & -2.48121582 & 1.68531370 \\
\hline $\mathrm{H}$ & 0.90193888 & -2.74038465 & -0.06847163 \\
\hline C & -1.31104399 & -4.45472182 & -0.51166547 \\
\hline $\mathrm{H}$ & -2.15549994 & -5.13512072 & -0.42600352 \\
\hline C & -0.88905786 & -4.15115549 & -1.77202238 \\
\hline $\mathrm{H}$ & 0.01240532 & -3.58142839 & -1.97380970 \\
\hline $\mathrm{O}$ & -1.50490741 & -4.69318968 & -2.85350035 \\
\hline C & -0.79330988 & -4.57394936 & -4.08099276 \\
\hline $\mathrm{H}$ & 0.15535248 & -5.12476740 & -4.04294583 \\
\hline $\mathrm{H}$ & -1.43191835 & -5.00647063 & -4.85319701 \\
\hline $\mathrm{H}$ & -0.59461800 & -3.52165008 & -4.32130329 \\
\hline $\mathrm{E}_{\text {tot }}^{\mathrm{a}}$ & & 005.7095977 & \\
\hline $\mathrm{ZPE}^{\mathrm{b}}$ & & 0.467715 & \\
\hline Imag & & -265.7807 & \\
\hline
\end{tabular}

${ }^{\mathrm{a}} E_{\text {tot }}$ : electronic energy. ${ }^{\mathrm{b}} \mathrm{ZPE}$ : zero point energy, unscaled

${ }^{\mathrm{C}}$ Imag: imaginary frequency 
TS16b; B3LYP/6-31G(d)

\begin{tabular}{|c|c|c|c|}
\hline & \multicolumn{3}{|c|}{ TS16b; B3LYP/6-31G(d) } \\
\hline $\mathrm{C}$ & 0.00000000 & 0.00000000 & 0.00000000 \\
\hline $\mathrm{O}$ & 1.21886437 & 0.00000000 & 0.00000000 \\
\hline $\mathrm{C}$ & -0.79825519 & 1.33129964 & 0.00000000 \\
\hline $\mathrm{H}$ & -0.07688827 & 2.04065007 & -0.42606970 \\
\hline $\mathrm{C}$ & -2.11108970 & 1.42507307 & -0.87883764 \\
\hline C & -2.10036087 & 0.71066230 & -2.25921339 \\
\hline $\mathrm{H}$ & -2.89796716 & 1.16904727 & -2.86347317 \\
\hline $\mathrm{H}$ & -1.15906354 & 0.92529304 & -2.78314237 \\
\hline $\mathrm{C}$ & -2.35038087 & -0.83651168 & -2.24287383 \\
\hline $\mathrm{C}$ & -1.08455048 & 1.81532290 & 1.44115790 \\
\hline $\mathrm{H}$ & -0.29920350 & 1.46858826 & 2.12006384 \\
\hline $\mathrm{H}$ & -1.07321991 & 2.91379378 & 1.46743614 \\
\hline $\mathrm{O}$ & -2.30390566 & 1.32073748 & 1.97229620 \\
\hline $\mathrm{C}$ & -3.49199216 & 1.75069630 & 1.27111526 \\
\hline $\mathrm{C}$ & -3.59399383 & 3.28978520 & 1.21737012 \\
\hline $\mathrm{H}$ & -4.60095905 & 3.57931566 & 0.89789925 \\
\hline $\mathrm{H}$ & -3.42021738 & 3.70908305 & 2.21436773 \\
\hline $\mathrm{H}$ & -2.88786530 & 3.75525191 & 0.52424365 \\
\hline C & -4.64186322 & 1.23689327 & 2.14593342 \\
\hline $\mathrm{H}$ & -4.63075704 & 1.76121035 & 3.10703720 \\
\hline $\mathrm{H}$ & -5.61101696 & 1.41431792 & 1.66708705 \\
\hline $\mathrm{H}$ & -4.54184837 & 0.16795522 & 2.34822999 \\
\hline $\mathrm{H}$ & -2.14834465 & 2.49322534 & -1.12972938 \\
\hline $\mathrm{C}$ & -3.47587389 & 1.13762346 & -0.16400117 \\
\hline $\mathrm{H}$ & -4.21621906 & 1.69731696 & -0.75657250 \\
\hline $\mathrm{C}$ & -3.94630632 & -0.33705220 & -0.19180235 \\
\hline $\mathrm{H}$ & -5.02885788 & -0.34867690 & -0.01432953 \\
\hline $\mathrm{H}$ & -3.52094094 & -0.88548743 & 0.65125319 \\
\hline C & -3.69258983 & -1.09197119 & -1.51752705 \\
\hline $\mathrm{H}$ & -4.48248560 & -0.80246505 & -2.22494499 \\
\hline $\mathrm{H}$ & -3.81806199 & -2.16915546 & -1.34941707 \\
\hline C & -2.45272826 & -1.34359819 & -3.69048409 \\
\hline $\mathrm{H}$ & -1.50582245 & -1.20516664 & -4.22662329 \\
\hline $\mathrm{H}$ & -2.69792042 & -2.41087541 & -3.71346571 \\
\hline $\mathrm{H}$ & -3.23239672 & -0.80592944 & -4.24369284 \\
\hline $\mathrm{H}$ & -0.20856783 & -1.18634829 & -2.17711180 \\
\hline C & -1.09212233 & -1.35913137 & -1.55711437 \\
\hline C & -0.90890735 & 2133 & -0.19988188 \\
\hline $\mathrm{H}$ & -1.79236950 & -1.10040983 & 0.41708441 \\
\hline $\mathrm{H}$ & 0.21547694 & -3.42710399 & -1.88719968 \\
\hline C & -0.80509603 & -3.78529752 & -1.82480444 \\
\hline $\mathrm{H}$ & -1.25300455 & -4.07081268 & -2.77162287 \\
\hline C & -1.33799522 & -4.23836892 & -0.64474936 \\
\hline $\mathrm{H}$ & -2.26509141 & -4.80909325 & -0.68876246 \\
\hline C & -0.88604493 & -3.88065720 & 0.65013190 \\
\hline $\mathrm{H}$ & -1.47969929 & -4.21874132 & 1.49402407 \\
\hline C & 0.13505486 & -2.97650606 & 0.87586362 \\
\hline $\mathrm{O}$ & 0.54132644 & -2.53719421 & 2.09073874 \\
\hline $\mathrm{H}$ & 0.89765748 & -2.74832374 & 0.14196254 \\
\hline C & -0.30905730 & -2.75908420 & 3.20737304 \\
\hline $\mathrm{H}$ & 0.15414280 & -2.23550623 & 4.04538518 \\
\hline $\mathrm{H}$ & -1.31251462 & -2.35188901 & 3.02901766 \\
\hline $\mathrm{H}$ & -0.38815734 & -3.82757543 & 3.44627809 \\
\hline $\mathrm{E}_{\text {tot }}{ }^{\mathrm{a}}$ & & 005.70795367 & \\
\hline $\mathrm{ZPE}^{\mathrm{b}}$ & & 0.468651 & \\
\hline Imag & & -294.2725 & \\
\hline
\end{tabular}

${ }^{\mathrm{a}} E_{\text {tot }}$ : electronic energy. ${ }^{\mathrm{b}} \mathrm{ZPE}$ : zero point energy, unscaled

${ }^{\mathrm{C}}$ Imag: imaginary frequency 
TS16c; B3LYP/6-31G(d)

\begin{tabular}{|c|c|c|c|}
\hline & \multicolumn{3}{|c|}{ TS16c; B3LYP/6-31G(d) } \\
\hline $\mathrm{C}$ & 0.00000000 & 0.00000000 & 0.00000000 \\
\hline $\mathrm{O}$ & 1.21614103 & 0.00000000 & 0.00000000 \\
\hline $\mathrm{C}$ & -0.78105993 & 1.34527132 & 0.00000000 \\
\hline $\mathrm{H}$ & -0.08929894 & 2.01338255 & -0.52965335 \\
\hline $\mathrm{C}$ & -2.17480933 & 1.46489660 & -0.73178952 \\
\hline C & -2.32386191 & 0.75853779 & -2.10483800 \\
\hline $\mathrm{H}$ & -3.17792613 & 1.22518520 & -2.62056215 \\
\hline $\mathrm{H}$ & -1.44143122 & 0.95990919 & -2.72670287 \\
\hline $\mathrm{C}$ & -2.57684134 & -0.78566559 & -2.04644800 \\
\hline $\mathrm{C}$ & -0.90515120 & 1.90122232 & 1.43694155 \\
\hline $\mathrm{H}$ & -0.04655849 & 1.59028502 & 2.03980963 \\
\hline $\mathrm{H}$ & -0.89963499 & 3.00014565 & 1.40616284 \\
\hline $\mathrm{O}$ & -2.05503359 & 1.42657552 & 2.11862169 \\
\hline $\mathrm{C}$ & -3.31762409 & 1.83305126 & 1.54718078 \\
\hline $\mathrm{C}$ & -3.44123486 & 3.37034507 & 1.48405120 \\
\hline $\mathrm{H}$ & -4.48199703 & 3.64715784 & 1.28379452 \\
\hline $\mathrm{H}$ & -3.15246204 & 3.80617276 & 2.44659975 \\
\hline $\mathrm{H}$ & -2.82835668 & 3.83054361 & 0.70398137 \\
\hline $\mathrm{C}$ & -4.35595874 & 1.32239981 & 2.55339357 \\
\hline $\mathrm{H}$ & -4.24006449 & 1.86120292 & 3.49933174 \\
\hline $\mathrm{H}$ & -5.37480747 & 1.48559233 & 2.18562728 \\
\hline $\mathrm{H}$ & -4.22224184 & 0.25776367 & 2.75846117 \\
\hline $\mathrm{H}$ & -2.21547999 & 2.53606753 & -0.97091868 \\
\hline $\mathrm{C}$ & -3.45886081 & 1.20348030 & 0.12560832 \\
\hline $\mathrm{H}$ & -4.24955442 & 1.76866666 & -0.39188741 \\
\hline $\mathrm{C}$ & -3.94971536 & -0.26436023 & 0.15448552 \\
\hline $\mathrm{H}$ & -5.00751654 & -0.26179941 & 0.44526153 \\
\hline $\mathrm{H}$ & -3.44596503 & -0.81814903 & 0.94955485 \\
\hline $\mathrm{C}$ & -3.84588866 & -1.01878793 & -1.19078274 \\
\hline $\mathrm{H}$ & -4.69694519 & -0.71012194 & -1.81439343 \\
\hline $\mathrm{H}$ & -3.97989700 & -2.09454046 & -1.01269838 \\
\hline C & -2.82096491 & -1.30988004 & -3.47142550 \\
\hline $\mathrm{H}$ & -1.93588378 & -1.16431600 & -4.10175368 \\
\hline $\mathrm{H}$ & -3.04375111 & -2.38247477 & -3.46166480 \\
\hline $\mathrm{H}$ & -3.66344866 & -0.79298635 & -3.94756989 \\
\hline $\mathrm{H}$ & -0.44362958 & -1.21560675 & -2.20109170 \\
\hline C & -1.26588320 & -1.31869013 & -1.48978019 \\
\hline C & -0.93399907 & -1.14880314 & -0.15711733 \\
\hline $\mathrm{H}$ & -1.75484814 & -1.09196973 & 0.54529210 \\
\hline C & 1.04623235 & -3.23701211 & -0.28629576 \\
\hline $\mathrm{H}$ & 2.07878245 & -2.89911223 & -0.25195797 \\
\hline C & 0.20861001 & -2.84801661 & 0.73104954 \\
\hline $\mathrm{H}$ & 0.61868069 & -2.33767456 & 1.59648299 \\
\hline $\mathrm{H}$ & -0.74409129 & -3.34335159 & 0.89362055 \\
\hline C & 0.62628825 & -3.88238370 & -1.48457268 \\
\hline $\mathrm{H}$ & 1.37090830 & -4.10520985 & -2.24550385 \\
\hline C & -0.67398197 & -4.14534423 & -1.79972747 \\
\hline $\mathrm{H}$ & -1.49326299 & -4.03262012 & -1.09541604 \\
\hline $\mathrm{O}$ & -0.99509713 & -4.68361834 & -3.00368821 \\
\hline C & -2.27383245 & -5.30129002 & -3.07469581 \\
\hline $\mathrm{H}$ & -2.31851157 & -6.19477808 & -2.43826833 \\
\hline $\mathrm{H}$ & -3.06980148 & -4.60555950 & -2.77593010 \\
\hline $\mathrm{H}$ & -2.42261589 & -5.59153804 & -4.11642398 \\
\hline $\mathrm{E}_{\text {tot }}^{\mathrm{a}}$ & & 005.70667560 & \\
\hline $\mathrm{ZPE}^{\mathrm{b}}$ & & 0.467783 & \\
\hline Imag & & -284.0726 & \\
\hline
\end{tabular}

${ }^{\mathrm{a}} E_{\text {tot }}$ : electronic energy. ${ }^{\mathrm{b}} \mathrm{ZPE}$ : zero point energy, unscaled

${ }^{\mathrm{c}}$ Imag: imaginary frequency 
TS16d; B3LYP/6-31G(d)

\begin{tabular}{|c|c|c|c|}
\hline & \multicolumn{3}{|c|}{ TS16d; B3LYP/6-31G(d) } \\
\hline $\mathrm{C}$ & 0.00000000 & 0.00000000 & 0.00000000 \\
\hline $\mathrm{O}$ & 1.22709653 & 0.00000000 & 0.00000000 \\
\hline $\mathrm{C}$ & -0.77645487 & 1.36577905 & 0.00000000 \\
\hline $\mathrm{H}$ & -1.04755576 & 1.57715081 & -1.04790693 \\
\hline $\mathrm{C}$ & -2.08286587 & 1.54989944 & 0.86932616 \\
\hline $\mathrm{C}$ & -3.36139564 & 0.72633754 & 0.58963552 \\
\hline $\mathrm{H}$ & -4.15760959 & 1.15519484 & 1.21679124 \\
\hline $\mathrm{H}$ & -3.68841978 & 0.85395202 & -0.45064995 \\
\hline $\mathrm{C}$ & -3.24993774 & -0.79522717 & 0.92197619 \\
\hline $\mathrm{C}$ & 0.19385137 & 2.45231149 & 0.48677378 \\
\hline $\mathrm{H}$ & 1.15801201 & 2.35269699 & -0.01193356 \\
\hline $\mathrm{H}$ & -0.21482002 & 3.45164247 & 0.27272338 \\
\hline $\mathrm{O}$ & 0.47440255 & 2.29506363 & 1.87352625 \\
\hline $\mathrm{C}$ & -0.63075829 & 2.44978125 & 2.78213828 \\
\hline $\mathrm{C}$ & -1.11092765 & 3.91706609 & 2.79759604 \\
\hline $\mathrm{H}$ & -1.80809387 & 4.08168926 & 3.62685820 \\
\hline $\mathrm{H}$ & -0.25063608 & 4.58051668 & 2.93454596 \\
\hline $\mathrm{H}$ & -1.62250367 & 4.21380502 & 1.87673561 \\
\hline $\mathrm{C}$ & -0.02349787 & 2.12743353 & 4.15214924 \\
\hline $\mathrm{H}$ & 0.75011783 & 2.86428731 & 4.39116097 \\
\hline $\mathrm{H}$ & -0.78488828 & 2.15783367 & 4.93969926 \\
\hline $\mathrm{H}$ & 0.44468341 & 1.13996546 & 4.15083567 \\
\hline $\mathrm{H}$ & -2.37466527 & 2.58402783 & 0.63781617 \\
\hline $\mathrm{C}$ & -1.80946047 & 1.49716756 & 2.39607455 \\
\hline $\mathrm{H}$ & -2.70796023 & 1.91438048 & 2.87718192 \\
\hline $\mathrm{C}$ & -1.68005780 & 0.03977429 & 2.89619979 \\
\hline $\mathrm{H}$ & -1.71208255 & 0.03474347 & 3.99244957 \\
\hline $\mathrm{H}$ & -0.69694405 & -0.35048847 & 2.62750623 \\
\hline C & -2.80769953 & -0.91106688 & 2.40657546 \\
\hline $\mathrm{H}$ & -3.70314284 & -0.71058185 & 3.01199941 \\
\hline $\mathrm{H}$ & -2.52544991 & -1.94863628 & 2.63255487 \\
\hline C & -4.61693458 & -1.46309490 & 0.72061687 \\
\hline $\mathrm{H}$ & -4.92935456 & -1.41978703 & -0.32911345 \\
\hline $\mathrm{H}$ & -4.59236882 & -2.51597100 & 1.02318419 \\
\hline $\mathrm{H}$ & -5.38605938 & -0.96225347 & 1.32118075 \\
\hline $\mathrm{H}$ & -2.36947948 & -0.66998176 & -1.04423382 \\
\hline C & -2.16773254 & -1.16977837 & -0.09420637 \\
\hline C & -0.82730139 & -1.18786646 & 0.23968988 \\
\hline $\mathrm{H}$ & -0.48953573 & -1.77489979 & 1.09367360 \\
\hline $\mathrm{H}$ & -1.94198625 & -3.70134934 & -0.19889893 \\
\hline C & -2.55864632 & -3.29898164 & -0.99440400 \\
\hline $\mathrm{H}$ & -3.62426540 & -3.45881926 & -0.87449610 \\
\hline C & -2.05494822 & -3.04706956 & -2.25070461 \\
\hline $\mathrm{H}$ & -2.77258812 & -2.86358492 & -3.04939822 \\
\hline C & -0.69273570 & -2.82714568 & -2.58356450 \\
\hline $\mathrm{H}$ & -0.44874521 & -2.48936548 & -3.58779611 \\
\hline C & 0.34237655 & -2.91440486 & -1.69189565 \\
\hline $\mathrm{O}$ & 1.58863395 & -2.59936675 & -2.06694739 \\
\hline $\mathrm{H}$ & 0.23119900 & -3.34949786 & -0.70348160 \\
\hline C & 2.59478450 & -2.69248311 & -1.04942210 \\
\hline $\mathrm{H}$ & 3.54415722 & -2.48843699 & -1.54685716 \\
\hline $\mathrm{H}$ & 2.40355491 & -1.94046024 & -0.27887485 \\
\hline $\mathrm{H}$ & 2.61840085 & -3.70329948 & -0.62179956 \\
\hline $\mathrm{E}_{\text {tot }}^{\mathrm{a}}$ & & 005.70950428 & \\
\hline $\mathrm{ZPE}^{\mathrm{b}}$ & & 0.467666 & \\
\hline $\operatorname{Imag}^{\mathrm{C}}$ & & -270.7906 & \\
\hline
\end{tabular}

${ }^{\mathrm{a}} E_{\text {tot }}:$ electronic energy. ${ }^{\mathrm{b}} \mathrm{ZPE}$ : zero point energy, unscaled

${ }^{\mathrm{c}}$ Imag: imaginary frequency 
TS22a; B3LYP/6-31G(d)

\begin{tabular}{|c|c|c|c|}
\hline & \multicolumn{3}{|c|}{ TS22a; B3LYP/6-31G(d) } \\
\hline $\mathrm{C}$ & 0.00000000 & 0.00000000 & 0.00000000 \\
\hline $\mathrm{H}$ & 1.09095144 & 0.00000000 & 0.00000000 \\
\hline $\mathrm{C}$ & -0.49875675 & 1.33634723 & 0.00000000 \\
\hline $\mathrm{H}$ & 0.21688950 & 2.14196249 & -0.13168449 \\
\hline $\mathrm{C}$ & -1.85566491 & 1.81849419 & 0.10686487 \\
\hline $\mathrm{O}$ & -2.10807777 & 3.00757131 & -0.16358545 \\
\hline $\mathrm{C}$ & -3.07856540 & 0.92614050 & 0.46463411 \\
\hline $\mathrm{H}$ & -3.46625825 & 0.58913604 & -0.51316099 \\
\hline $\mathrm{C}$ & -2.93509544 & -0.33412853 & 1.35410272 \\
\hline $\mathrm{C}$ & -1.97770623 & -1.42527453 & 0.85577827 \\
\hline $\mathrm{H}$ & -2.17519448 & -2.33127568 & 1.44743862 \\
\hline $\mathrm{H}$ & -2.22055405 & -1.68753962 & -0.18234557 \\
\hline $\mathrm{C}$ & -0.48136120 & -1.09594254 & 0.98826044 \\
\hline $\mathrm{C}$ & -4.16760979 & 1.80199881 & 1.10599302 \\
\hline $\mathrm{H}$ & -4.25137009 & 2.74717203 & 0.57143101 \\
\hline $\mathrm{H}$ & -5.13549540 & 1.27883753 & 1.06265608 \\
\hline $\mathrm{O}$ & -3.83497308 & 2.15028159 & 2.44352340 \\
\hline $\mathrm{C}$ & -3.69178114 & 1.05704282 & 3.36793042 \\
\hline C & -5.05384101 & 0.37486192 & 3.61777858 \\
\hline $\mathrm{H}$ & -4.98201880 & -0.31987817 & 4.46229433 \\
\hline $\mathrm{H}$ & -5.80280804 & 1.13551637 & 3.86188227 \\
\hline $\mathrm{H}$ & -5.41866192 & -0.19319562 & 2.75698374 \\
\hline $\mathrm{C}$ & -3.24801796 & 1.73455779 & 4.66957886 \\
\hline $\mathrm{H}$ & -4.05044296 & 2.38192702 & 5.03813365 \\
\hline $\mathrm{H}$ & -3.01883658 & 0.99328773 & 5.44368172 \\
\hline $\mathrm{H}$ & -2.36616334 & 2.35794306 & 4.50341668 \\
\hline $\mathrm{H}$ & -3.93217199 & -0.79557507 & 1.32269126 \\
\hline C & -2.64537934 & 0.02851642 & 2.83475233 \\
\hline $\mathrm{H}$ & -2.78252235 & -0.89439381 & 3.42143758 \\
\hline C & -1.17476625 & 0.45480975 & 3.00229230 \\
\hline $\mathrm{H}$ & -0.94220567 & 0.58694212 & 4.06546233 \\
\hline $\mathrm{H}$ & -1.00334995 & 1.42505129 & 2.52708033 \\
\hline C & -0.20796713 & -0.59996228 & 2.44388789 \\
\hline $\mathrm{H}$ & -0.26190095 & -1.48445924 & 3.09577824 \\
\hline $\mathrm{H}$ & 0.82494887 & -0.23349046 & 2.51442630 \\
\hline C & 0.35112480 & -2.38343134 & 0.80189816 \\
\hline $\mathrm{H}$ & 0.16528608 & -2.88127577 & -0.15410285 \\
\hline $\mathrm{H}$ & 1.42546803 & -2.17051859 & 0.86649779 \\
\hline $\mathrm{H}$ & 0.10948270 & -3.10493672 & 1.59047567 \\
\hline $\mathrm{H}$ & 1.84745226 & 1.45284599 & -3.38341138 \\
\hline C & 0.89677820 & 1.13418952 & -2.96203646 \\
\hline C & -0.03585518 & 2.12624961 & -2.72603180 \\
\hline $\mathrm{H}$ & -1.06644236 & 1.93135667 & -2.45225125 \\
\hline C & 0.73619460 & -0.19854478 & -2.57176080 \\
\hline $\mathrm{H}$ & 1.59087722 & -0.85448771 & -2.72913651 \\
\hline C & -0.32374358 & -0.69389826 & -1.77557506 \\
\hline $\mathrm{H}$ & -1.31468004 & -0.27851509 & -1.93445146 \\
\hline $\mathrm{H}$ & -0.35723242 & -1.77563457 & -1.68538873 \\
\hline $\mathrm{O}$ & 0.30462205 & 3.39788036 & -2.93555374 \\
\hline C & -0.72166251 & 4.38936945 & -2.72464680 \\
\hline $\mathrm{H}$ & -0.22671277 & 5.35370944 & -2.84523178 \\
\hline $\mathrm{H}$ & -1.50678495 & 4.28225709 & -3.48269326 \\
\hline $\mathrm{H}$ & -1.14241943 & 4.28227430 & -1.72230840 \\
\hline $\mathrm{E}_{\text {tot }}{ }^{\mathrm{a}}$ & & 005.74719271 & \\
\hline $\mathrm{ZPE}$ & & 0.469648 & \\
\hline $\operatorname{Imag}^{c}$ & & -392.3063 & \\
\hline
\end{tabular}

${ }^{\mathrm{a}} E_{\text {tot: }}$ : electronic energy. ${ }^{\mathrm{b}} \mathrm{ZPE}$ : zero point energy, unscaled

${ }^{\mathrm{c}}$ Imag: imaginary frequency 
TS22b; B3LYP/6-31G(d)

\begin{tabular}{|c|c|c|c|}
\hline & \multicolumn{3}{|c|}{ TS22b; B3LYP/6-31G(d) } \\
\hline $\mathrm{C}$ & 0.00000000 & 0.00000000 & 0.00000000 \\
\hline $\mathrm{H}$ & 1.08788738 & 0.00000000 & 0.00000000 \\
\hline $\mathrm{C}$ & -0.57803986 & 1.30310692 & 0.00000000 \\
\hline $\mathrm{H}$ & 0.16209329 & 2.09569235 & -0.06093617 \\
\hline $\mathrm{C}$ & -1.67088382 & 1.80780373 & 0.90786358 \\
\hline $\mathrm{O}$ & -1.52831461 & 2.91993468 & 1.39427292 \\
\hline $\mathrm{C}$ & -2.99140854 & 1.04332405 & 1.10756313 \\
\hline $\mathrm{H}$ & -3.49364189 & 1.08752661 & 0.12749928 \\
\hline $\mathrm{C}$ & -2.91854126 & -0.45039980 & 1.50567133 \\
\hline $\mathrm{C}$ & -2.11346650 & -1.36109834 & 0.56374156 \\
\hline $\mathrm{H}$ & -2.38962900 & -2.39662701 & 0.80768276 \\
\hline $\mathrm{H}$ & -2.43974092 & -1.20425609 & -0.47462236 \\
\hline $\mathrm{C}$ & -0.57391757 & -1.25909021 & 0.65545811 \\
\hline $\mathrm{C}$ & -3.88405756 & 1.77078125 & 2.12333462 \\
\hline $\mathrm{H}$ & -3.86199106 & 2.84714259 & 1.94862906 \\
\hline $\mathrm{H}$ & -4.92133346 & 1.41828853 & 2.00837036 \\
\hline $\mathrm{O}$ & -3.43097295 & 1.59828193 & 3.45638130 \\
\hline $\mathrm{C}$ & -3.35722298 & 0.24254161 & 3.93188544 \\
\hline $\mathrm{C}$ & -4.76907219 & -0.36646197 & 4.06762436 \\
\hline $\mathrm{H}$ & -4.72049064 & -1.32498947 & 4.59651062 \\
\hline $\mathrm{H}$ & -5.40316552 & 0.31272548 & 4.64700312 \\
\hline $\mathrm{H}$ & -5.26016569 & -0.54733011 & 3.10693525 \\
\hline $\mathrm{C}$ & -2.75646476 & 0.38023174 & 5.33538052 \\
\hline $\mathrm{H}$ & -3.46087702 & 0.90835279 & 5.98618757 \\
\hline $\mathrm{H}$ & -2.54933499 & -0.60207067 & 5.77518105 \\
\hline $\mathrm{H}$ & -1.83058953 & 0.95936535 & 5.30704870 \\
\hline $\mathrm{H}$ & -3.95619998 & -0.80329140 & 1.43432997 \\
\hline $\mathrm{C}$ & -2.46634677 & -0.61464690 & 2.97934144 \\
\hline $\mathrm{H}$ & -2.63293403 & -1.66875036 & 3.25358659 \\
\hline C & -0.95254143 & -0.35233708 & 3.09406258 \\
\hline $\mathrm{H}$ & -0.61512762 & -0.53748751 & 4.11993840 \\
\hline $\mathrm{H}$ & -0.72929007 & 0.70053804 & 2.89168198 \\
\hline C & -0.15940892 & -1.28024007 & 2.16480529 \\
\hline $\mathrm{H}$ & -0.29375876 & -2.31040990 & 2.52736787 \\
\hline $\mathrm{H}$ & 0.91454900 & -1.06714732 & 2.24488387 \\
\hline C & 0.03131836 & -2.53380634 & 0.02506320 \\
\hline $\mathrm{H}$ & -0.30052142 & -2.69338277 & -1.00333194 \\
\hline $\mathrm{H}$ & 1.12586630 & -2.49270252 & 0.01096954 \\
\hline $\mathrm{H}$ & -0.26489448 & -3.41260369 & 0.60991527 \\
\hline $\mathrm{H}$ & -0.12539606 & 2.90254262 & -2.89104532 \\
\hline C & -0.42263272 & 1.89419259 & -2.61127627 \\
\hline C & -1.50138663 & 1.73270654 & -1.72281379 \\
\hline $\mathrm{H}$ & -2.08529240 & 0.81716232 & -1.76852801 \\
\hline C & 0.45932720 & 0.85439558 & -2.88489266 \\
\hline $\mathrm{H}$ & 1.39240209 & 1.09009112 & -3.38860763 \\
\hline C & 0.27027701 & -0.43570115 & -2.38672924 \\
\hline $\mathrm{H}$ & -0.72265592 & -0.83738391 & -2.23516060 \\
\hline $\mathrm{O}$ & 1.15990488 & -1.45331362 & -2.55737682 \\
\hline $\mathrm{H}$ & -2.11110959 & 2.61055825 & -1.51686191 \\
\hline C & 2.50763951 & -1.12587813 & -2.86251881 \\
\hline $\mathrm{H}$ & 2.90595000 & -0.37912012 & -2.16341975 \\
\hline $\mathrm{H}$ & 3.07275191 & -2.05521371 & -2.76845928 \\
\hline $\mathrm{H}$ & 2.60826881 & -0.74682778 & -3.88790892 \\
\hline $\mathrm{E}_{\text {tot }}{ }^{\mathrm{a}}$ & & 1005.73678180 & \\
\hline $\mathrm{ZPE}^{\mathrm{b}}$ & & 0.469909 & \\
\hline $\operatorname{Imag}^{c}$ & & -528.3014 & \\
\hline
\end{tabular}

${ }^{\mathrm{a}} E_{\text {tot }}$ : electronic energy. ${ }^{\mathrm{b}} \mathrm{ZPE}$ : zero point energy, unscaled

${ }^{\mathrm{C}}$ Imag: imaginary frequency 
TS11; B3LYP/6-31G(d)

\begin{tabular}{|c|c|c|c|}
\hline & \multicolumn{3}{|c|}{ TS11; B3LYP/6-31G(d) } \\
\hline $\mathrm{C}$ & 0.00000000 & 0.00000000 & 0.00000000 \\
\hline $\mathrm{O}$ & 1.21719301 & 0.00000000 & 0.00000000 \\
\hline $\mathrm{C}$ & -0.78306084 & 1.34510652 & 0.00000000 \\
\hline $\mathrm{H}$ & -0.10068077 & 2.01048428 & -0.54555191 \\
\hline $\mathrm{C}$ & -2.19008281 & 1.45502803 & -0.71041825 \\
\hline C & -2.36336780 & 0.74904427 & -2.08151758 \\
\hline $\mathrm{H}$ & -3.23310806 & 1.20836639 & -2.57640972 \\
\hline $\mathrm{H}$ & -1.49779934 & 0.96046617 & -2.72366548 \\
\hline $\mathrm{C}$ & -2.60448552 & -0.79802448 & -2.02111276 \\
\hline $\mathrm{C}$ & -0.88801919 & 1.91192015 & 1.43378458 \\
\hline $\mathrm{H}$ & -0.01474131 & 1.61634404 & 2.02308280 \\
\hline $\mathrm{H}$ & -0.89806634 & 3.01035729 & 1.39418013 \\
\hline $\mathrm{O}$ & -2.02008194 & 1.42754847 & 2.13781509 \\
\hline C & -3.29617600 & 1.82126407 & 1.58712041 \\
\hline $\mathrm{C}$ & -3.43183034 & 3.35794835 & 1.52139385 \\
\hline $\mathrm{H}$ & -4.47679360 & 3.62744124 & 1.33350868 \\
\hline $\mathrm{H}$ & -3.13387505 & 3.79863119 & 2.47893827 \\
\hline $\mathrm{H}$ & -2.83146329 & 3.82063426 & 0.73294834 \\
\hline $\mathrm{C}$ & -4.31268721 & 1.30530626 & 2.61246420 \\
\hline $\mathrm{H}$ & -4.18774363 & 1.85031642 & 3.55367253 \\
\hline $\mathrm{H}$ & -5.33917560 & 1.45488658 & 2.26064699 \\
\hline $\mathrm{H}$ & -4.16378103 & 0.24332982 & 2.82074933 \\
\hline $\mathrm{H}$ & 72715 & 2.52579670 & -0.95001452 \\
\hline $\mathrm{C}$ & -3.45811581 & 1.18694829 & 0.16942784 \\
\hline $\mathrm{H}$ & -4.26068211 & 1.74683052 & -0.33517845 \\
\hline $\mathrm{C}$ & -3.94021052 & -0.28509538 & 0.20792275 \\
\hline $\mathrm{H}$ & -4.99379676 & -0.28784202 & 0.51332619 \\
\hline $\mathrm{H}$ & -3.42299993 & -0.83431791 & 0.99772490 \\
\hline $\mathrm{C}$ & -3.85150303 & -1.04341925 & -1.13773488 \\
\hline $\mathrm{H}$ & -4.71804557 & 51168 & -1.74564303 \\
\hline $\mathrm{H}$ & -3.97067730 & 973915 & 157117 \\
\hline C & -2.87295386 & -1.33251158 & -3.43743332 \\
\hline $\mathrm{H}$ & -1.99988619 & -1.18824281 & -4.08498418 \\
\hline $\mathrm{H}$ & -3.09542576 & -2.40538477 & -3.41254834 \\
\hline $\mathrm{H}$ & -3.72450543 & -0.82007823 & -3.90166929 \\
\hline $\mathrm{H}$ & -0.46589097 & -1.16594993 & -2.20680639 \\
\hline C & -1.27473604 & -1.30387653 & -1.48720826 \\
\hline C & -0.93444288 & -1.14308784 & -0.15849822 \\
\hline $\mathrm{H}$ & -1.74694200 & -1.09448003 & 0.55271421 \\
\hline $\mathrm{H}$ & -1.66760722 & -3.86504051 & -1.07319662 \\
\hline C & -0.88933646 & -3.95391769 & -1.82125827 \\
\hline $\mathrm{H}$ & -1.21471027 & -4.27591990 & -2.80633784 \\
\hline C & 0.43599169 & -3.88323523 & -1.51889283 \\
\hline $\mathrm{H}$ & 1.14985613 & -4.10080934 & -2.31394838 \\
\hline C & 0.98315424 & -3.36894236 & -0.29517218 \\
\hline C & 0.15671566 & -2.96504217 & 0.72394474 \\
\hline $\mathrm{H}$ & -0.84553607 & -3.36660208 & 0.82293577 \\
\hline $\mathrm{H}$ & 0.57309806 & -2.52184496 & 1.62379048 \\
\hline C & 2.46586198 & -3.07616728 & -0.25815095 \\
\hline $\mathrm{H}$ & 2.70298693 & -2.18357949 & -0.85005752 \\
\hline $\mathrm{H}$ & 2.81375559 & -2.88813430 & 0.76142792 \\
\hline $\mathrm{H}$ & 3.04181017 & -3.91503392 & -0.66857172 \\
\hline $\mathrm{E}_{\text {tot }}^{\mathrm{a}}$ & & 30.502065673 & \\
\hline $\mathrm{ZPE}^{\mathrm{b}}$ & & 0.462503 & \\
\hline $\operatorname{Imag}^{c}$ & & -286.2002 & \\
\hline
\end{tabular}

${ }^{a} E_{\text {tot }}$ : electronic energy. ${ }^{b} Z P E$ : zero point energy, unscaled.

${ }^{\mathrm{c}}$ Imag: imaginary frequency 
TS12; B3LYP/6-31G(d)

\begin{tabular}{|c|c|c|c|}
\hline & \multicolumn{3}{|c|}{ TS12; B3LYP/6-31G(d) } \\
\hline $\mathrm{C}$ & 0.00000000 & 0.00000000 & 0.00000000 \\
\hline $\mathrm{O}$ & 1.21586641 & 0.00000000 & 0.00000000 \\
\hline $\mathrm{C}$ & -0.78452429 & 1.34452487 & 0.00000000 \\
\hline $\mathrm{H}$ & -0.09567906 & 2.01306468 & -0.53313196 \\
\hline $\mathrm{C}$ & -2.18153438 & 1.45420034 & -0.73101566 \\
\hline C & -2.33845644 & 0.73910086 & -2.10080108 \\
\hline $\mathrm{H}$ & -3.19972827 & 1.19950243 & -2.60936324 \\
\hline $\mathrm{H}$ & -1.46408797 & 0.94439240 & -2.73310654 \\
\hline $\mathrm{C}$ & -2.58718463 & -0.80621299 & -2.03724825 \\
\hline $\mathrm{C}$ & -0.90814519 & 1.90466172 & 1.43547018 \\
\hline $\mathrm{H}$ & -0.04765576 & 1.59680540 & 2.03723873 \\
\hline $\mathrm{H}$ & -0.90567455 & 3.00326703 & 1.40217837 \\
\hline $\mathrm{O}$ & -2.05589295 & 1.42997575 & 2.12022348 \\
\hline C & -3.31945899 & 1.83506274 & 1.54821878 \\
\hline $\mathrm{C}$ & -3.43846398 & 3.37223753 & 1.47268562 \\
\hline $\mathrm{H}$ & -4.47793478 & 3.65060662 & 1.26839122 \\
\hline $\mathrm{H}$ & -3.15009702 & 3.81416975 & 2.43252380 \\
\hline $\mathrm{H}$ & -2.82208658 & 3.82443852 & 0.69076168 \\
\hline $\mathrm{C}$ & -4.35786493 & 1.33430813 & 2.55894333 \\
\hline $\mathrm{H}$ & -4.23926949 & 1.87833756 & 3.50155782 \\
\hline $\mathrm{H}$ & -5.37657066 & 1.49838396 & 2.19121268 \\
\hline $\mathrm{H}$ & -4.22804488 & 0.27043136 & 2.77023556 \\
\hline $\mathrm{H}$ & -2.22678213 & 2.52325995 & -0.97824926 \\
\hline $\mathrm{C}$ & -3.46180000 & 1.19241320 & 0.13216986 \\
\hline $\mathrm{H}$ & -4.25784282 & 1.74729495 & -0.38823175 \\
\hline $\mathrm{C}$ & -3.94204153 & -0.27848439 & 0.17775512 \\
\hline $\mathrm{H}$ & -4.99861238 & -0.28061020 & 0.47252410 \\
\hline $\mathrm{H}$ & -3.43117403 & -0.82013401 & 0.97717030 \\
\hline $\mathrm{C}$ & -3.84014463 & -1.04819521 & -1.16052541 \\
\hline $\mathrm{H}$ & -4.70295051 & -0.76192786 & -1.77814084 \\
\hline $\mathrm{H}$ & -3.95492654 & -2.12346245 & -0.96933478 \\
\hline C & -2.84785644 & -1.34125913 & -3.45497171 \\
\hline $\mathrm{H}$ & -1.97074651 & -1.19823635 & -4.09742515 \\
\hline $\mathrm{H}$ & -3.07184205 & -2.41366035 & -3.43127988 \\
\hline $\mathrm{H}$ & -3.69588863 & -0.82700489 & -3.92374824 \\
\hline $\mathrm{H}$ & -0.44730426 & -1.18726968 & -2.20533055 \\
\hline C & -1.26357042 & -1.31547939 & -1.49232766 \\
\hline C & -0.93515061 & -1.14432932 & -0.16226001 \\
\hline $\mathrm{H}$ & -1.75619453 & -1.09465169 & 0.53918103 \\
\hline C & 0.98711315 & -3.34954732 & -0.35463236 \\
\hline $\mathrm{H}$ & 2.04515614 & -3.09452764 & -0.33006855 \\
\hline C & 0.20784316 & -2.94663243 & 0.69575313 \\
\hline $\mathrm{H}$ & 0.66554065 & -2.48052778 & 1.56259535 \\
\hline $\mathrm{H}$ & -0.78494308 & -3.35489809 & 0.85338852 \\
\hline C & 0.47473227 & -3.86534186 & -1.59194077 \\
\hline C & -0.86694400 & -3.95297650 & -1.83061487 \\
\hline $\mathrm{H}$ & -1.60492103 & -3.88055273 & -1.04219383 \\
\hline $\mathrm{H}$ & -1.23866771 & -4.27490591 & -2.79958322 \\
\hline C & 1.47362110 & -4.15615856 & -2.69290023 \\
\hline $\mathrm{H}$ & 2.14076712 & -4.98043033 & -2.40905735 \\
\hline $\mathrm{H}$ & 0.97626815 & -4.43676499 & -3.62640446 \\
\hline $\mathrm{H}$ & 2.11218500 & -3.28657684 & -2.89464469 \\
\hline $\mathrm{E}_{\text {tot }}^{\mathrm{a}}$ & & 30.501293876 & \\
\hline $\mathrm{ZPE}^{\mathrm{b}}$ & & 0.462658 & \\
\hline $\operatorname{Imag}^{c}$ & & -284.9104 & \\
\hline
\end{tabular}

${ }^{\mathrm{a}} E_{\text {tot: }}$ electronic energy. ${ }^{\mathrm{b}} \mathrm{ZPE}$ : zero point energy, unscaled.

${ }^{\mathrm{c}}$ Imag: imaginary frequency 
23a; UB3LYP/6-31G(d)

\begin{tabular}{l|ccc} 
& \multicolumn{3}{|c}{ 23a; UB3LYP/6-31G(d) } \\
\hline $\mathrm{C}$ & 0.00000000 & 0.00000000 & 0.00000000 \\
$\mathrm{H}$ & 1.09531160 & 0.00000000 & 0.00000000 \\
$\mathrm{C}$ & -0.63710792 & 1.30835663 & 0.00000000 \\
$\mathrm{H}$ & -0.84325711 & 1.81853760 & 0.94252512 \\
$\mathrm{C}$ & -1.02223318 & 2.06983937 & -1.16984669 \\
$\mathrm{O}$ & -1.35633959 & 3.25382631 & -1.02384301 \\
$\mathrm{C}$ & -1.02151854 & 1.43934549 & -2.58584400 \\
$\mathrm{H}$ & -0.00178661 & 1.59317890 & -2.97705063 \\
$\mathrm{C}$ & -1.37042563 & -0.06846172 & -2.78758761 \\
$\mathrm{C}$ & -0.46309857 & -1.12210730 & -2.12828492 \\
$\mathrm{H}$ & -0.70965696 & -2.09751297 & -2.57629670 \\
$\mathrm{H}$ & 0.58971645 & -0.92673836 & -2.37478239 \\
$\mathrm{C}$ & -0.62399359 & -1.24576570 & -0.58669271 \\
$\mathrm{C}$ & -1.99854503 & 2.23370191 & -3.46660831 \\
$\mathrm{H}$ & -1.86342546 & 3.30482031 & -3.31714170 \\
$\mathrm{H}$ & -1.81893943 & 1.99841987 & -4.52645717 \\
$\mathrm{O}$ & -3.34594350 & 1.96885034 & -3.09877671 \\
$\mathrm{C}$ & -3.79737176 & 0.61019160 & -3.25294988 \\
$\mathrm{C}$ & -3.86486150 & 0.22915782 & -4.74705775 \\
$\mathrm{H}$ & -4.39752315 & -0.72002048 & -4.87367747 \\
$\mathrm{H}$ & -4.40946116 & 1.00442031 & -5.29585926 \\
$\mathrm{H}$ & -2.88025491 & 0.11629158 & -5.21081826 \\
$\mathrm{C}$ & -5.22536407 & 0.63564723 & -2.69664066 \\
$\mathrm{H}$ & -5.85111421 & 1.27856913 & -3.32391565 \\
$\mathrm{H}$ & -5.66342670 & -0.36886611 & -2.68575195 \\
$\mathrm{H}$ & -5.24264885 & 1.04076751 & -1.68185921 \\
$\mathrm{H}$ & -1.22911373 & -0.21644027 & -3.86717831 \\
$\mathrm{C}$ & -2.86048971 & -0.36997182 & -2.47653328 \\
$\mathrm{H}$ & -3.06608413 & -1.37724502 & -2.87296282 \\
$\mathrm{C}$ & -3.09577901 & -0.43945450 & -0.95360498 \\
$\mathrm{H}$ & -4.11705180 & -0.78199693 & -0.75179366 \\
$\mathrm{H}$ & -3.02450059 & 0.56009269 & -0.51158809 \\
$\mathrm{C}$ & -2.12915333 & -1.41721218 & -0.25660278 \\
$\mathrm{H}$ & -2.40721814 & -2.43856271 & -0.55501847 \\
$\mathrm{H}$ & -2.27193986 & -1.36832169 & 0.83064310 \\
$\mathrm{C}$ & 0.15387192 & -2.47668499 & -0.08352723 \\
$\mathrm{H}$ & 1.22332235 & -2.38927120 & -0.31120202 \\
$\mathrm{H}$ & 0.04974284 & -2.58915569 & 1.00172755 \\
$\mathrm{H}$ & -0.21654588 & -3.39318213 & -0.55758135 \\
\hline $\mathrm{E}$ at & & -735.180140895 & \\
$\mathrm{ZPE}{ }^{\mathrm{b}}$ & & 0.343815 & \\
$\mathrm{Imag}$ & & -- & \\
\hline $\mathrm{d}$ & & & \\
\hline
\end{tabular}

${ }^{\mathrm{a}} E_{\text {tot }}$ : electronic energy. ${ }^{\mathrm{b}} \mathrm{ZPE}$ : zero point energy, unscaled

${ }^{\mathrm{c}}$ Imag: imaginary frequency 


\begin{tabular}{l|ccc} 
& \multicolumn{3}{|c}{ 23b; UB3LYP/6-31G(d) } \\
\hline $\mathrm{C}$ & 0.00000000 & 0.00000000 & 0.00000000 \\
$\mathrm{H}$ & 1.09263589 & 0.00000000 & 0.00000000 \\
$\mathrm{C}$ & -0.63768696 & 1.30910719 & 0.00000000 \\
$\mathrm{H}$ & -0.69996567 & 1.88895921 & -0.92292354 \\
$\mathrm{C}$ & -1.15248825 & 2.00238925 & 1.16220789 \\
$\mathrm{O}$ & -1.30528456 & 3.23215264 & 1.11207029 \\
$\mathrm{C}$ & -1.72047102 & 1.27115095 & 2.40015934 \\
$\mathrm{H}$ & -2.77578309 & 1.56991579 & 2.30839039 \\
$\mathrm{C}$ & -1.75627228 & -0.28713966 & 2.52606907 \\
$\mathrm{C}$ & -1.96994622 & -1.03305254 & 1.18737066 \\
$\mathrm{H}$ & -2.42671498 & -2.00885687 & 1.40480613 \\
$\mathrm{H}$ & -2.69751912 & -0.48558494 & 0.57221567 \\
$\mathrm{C}$ & -0.68010065 & -1.29925708 & 0.37396270 \\
$\mathrm{C}$ & -1.22866109 & 1.92334128 & 3.71037197 \\
$\mathrm{H}$ & -0.93046828 & 2.95560199 & 3.51467760 \\
$\mathrm{H}$ & -2.04913738 & 1.94710701 & 4.44102977 \\
$\mathrm{O}$ & -0.09628538 & 1.27336422 & 4.26108057 \\
$\mathrm{C}$ & -0.31209717 & -0.09748422 & 4.64957478 \\
$\mathrm{C}$ & -1.44887059 & -0.22457080 & 5.68639237 \\
$\mathrm{H}$ & -1.43514444 & -1.22763960 & 6.12660584 \\
$\mathrm{H}$ & -1.29902109 & 0.50277596 & 6.49155310 \\
$\mathrm{H}$ & -2.44701836 & -0.06718435 & 5.26790588 \\
$\mathrm{C}$ & 0.99960409 & -0.49268275 & 5.33811730 \\
$\mathrm{H}$ & 1.10678391 & 0.07306494 & 6.26927746 \\
$\mathrm{H}$ & 1.01484271 & -1.56126845 & 5.57986446 \\
$\mathrm{H}$ & 1.86070910 & -0.26095384 & 4.70680189 \\
$\mathrm{H}$ & -2.67489983 & -0.46427766 & 3.10032982 \\
$\mathrm{C}$ & -0.61902936 & -0.94393680 & 3.37518073 \\
$\mathrm{H}$ & -1.03127395 & -1.90478477 & 3.72276607 \\
$\mathrm{C}$ & 0.63729904 & -1.28924425 & 2.55535572 \\
$\mathrm{H}$ & 1.32138347 & -1.88688734 & 3.16730756 \\
$\mathrm{H}$ & 1.17612193 & -0.36856198 & 2.29880784 \\
$\mathrm{C}$ & 0.30023494 & -2.07638986 & 1.28226489 \\
$\mathrm{H}$ & -0.15025368 & -3.04334667 & 1.55064971 \\
$\mathrm{H}$ & 1.21966579 & -2.30258155 & 0.72507491 \\
$\mathrm{C}$ & -1.01819813 & -2.12561761 & -0.88701334 \\
$\mathrm{H}$ & -1.71897754 & -1.58704817 & -1.53527295 \\
$\mathrm{H}$ & -0.11483841 & -2.33799063 & -1.46999611 \\
$\mathrm{H}$ & -1.47780782 & -3.08375848 & -0.61169268 \\
\hline $\mathrm{E}_{\text {tot }}^{\mathrm{a}}$ & & -735.172669974 & \\
$\mathrm{ZPE} \mathrm{b}$ & & 0.343708 & \\
$\mathrm{Imag}$ & & -- & \\
\hline $\mathrm{a}$ & & & \\
$\mathrm{E}$ & & & \\
$\mathrm{H}$ & & & \\
$\mathrm{H}$ & &
\end{tabular}

\footnotetext{
${ }^{a} E_{\text {tot }}$ : electronic energy. ${ }^{b} \mathrm{ZPE}$ : zero point energy, unscaled

${ }^{\mathrm{c}}$ Imag: imaginary frequency
} 


\begin{tabular}{l|ccc} 
& \multicolumn{3}{|c}{ cis 24 ; B 3 LP/6-31G(d) } \\
\hline $\mathrm{C}$ & 0.00000000 & 0.00000000 & 0.00000000 \\
$\mathrm{H}$ & 1.09184774 & 0.00000000 & 0.00000000 \\
$\mathrm{C}$ & -0.56864413 & 1.21835074 & 0.00000000 \\
$\mathrm{H}$ & 0.08746957 & 2.08568214 & -0.04201365 \\
$\mathrm{C}$ & -1.98158654 & 1.63892293 & -0.02252554 \\
$\mathrm{O}$ & -2.21259850 & 2.82405565 & -0.25901774 \\
$\mathrm{C}$ & -3.23973546 & 0.73728009 & 0.12641536 \\
$\mathrm{C}$ & -3.09889067 & -0.76985127 & 0.42185168 \\
$\mathrm{C}$ & -2.04640833 & -1.47177598 & -0.45375401 \\
$\mathrm{H}$ & -2.30669657 & -2.53788268 & -0.50714177 \\
$\mathrm{H}$ & -2.11771467 & -1.08339431 & -1.47627559 \\
$\mathrm{C}$ & -0.59186678 & -1.38514689 & 0.05012740 \\
$\mathrm{C}$ & -4.20821895 & 1.36718928 & 1.15007740 \\
$\mathrm{H}$ & -4.16842960 & 2.45636161 & 1.07487176 \\
$\mathrm{H}$ & -5.22043830 & 1.03862343 & 0.87874634 \\
$\mathrm{O}$ & -3.89000000 & 1.04473050 & 2.49083743 \\
$\mathrm{C}$ & -3.97834338 & -0.35980171 & 2.80843490 \\
$\mathrm{C}$ & -5.41243051 & -0.89280909 & 2.60606685 \\
$\mathrm{H}$ & -5.50826493 & -1.88204481 & 3.06695114 \\
$\mathrm{H}$ & -6.12717606 & -0.21979135 & 3.09138714 \\
$\mathrm{H}$ & -5.69960818 & -0.99373571 & 1.55574410 \\
$\mathrm{C}$ & -3.65728488 & -0.41564521 & 4.30647625 \\
$\mathrm{H}$ & -4.45668057 & 0.07794745 & 4.86843727 \\
$\mathrm{H}$ & -3.57839080 & -1.45079441 & 4.65677664 \\
$\mathrm{H}$ & -2.72289564 & 0.10531675 & 4.52866337 \\
$\mathrm{H}$ & -4.06849262 & -1.15880221 & 0.08679462 \\
$\mathrm{C}$ & -2.95907336 & -1.14647288 & 1.92930097 \\
$\mathrm{H}$ & -3.23677849 & -2.21049373 & 1.99536582 \\
$\mathrm{C}$ & -1.50470079 & -1.04724930 & 2.42585770 \\
$\mathrm{H}$ & -1.43429432 & -1.44079344 & 3.44520292 \\
$\mathrm{H}$ & -1.19344065 & 0.00279974 & 2.47501438 \\
$\mathrm{C}$ & -0.54651579 & -1.84270789 & 1.53452620 \\
$\mathrm{H}$ & -0.81485325 & -2.90923154 & 1.56592163 \\
$\mathrm{H}$ & 0.47989688 & -1.76799058 & 1.91772097 \\
$\mathrm{C}$ & 0.29294957 & -2.31717662 & -0.80457362 \\
$\mathrm{H}$ & 0.28896900 & -2.01280284 & -1.85774690 \\
$\mathrm{H}$ & 1.33221074 & -2.31065172 & -0.45420873 \\
$\mathrm{H}$ & -0.07296667 & -3.34873898 & -0.75006845 \\
$\mathrm{O}$ & -3.85840822 & 0.81100439 & -1.17391805 \\
$\mathrm{H}$ & -3.84064185 & 1.75321351 & -1.41946383 \\
\hline $\mathrm{E}$ tot ${ }^{\mathrm{a}}$ & & -810.485563645 & \\
$\mathrm{ZPE}$ & & 0.352435 & \\
$\mathrm{Imag}$ & & -- & \\
\hline $\mathrm{E}$ & & &
\end{tabular}

${ }^{\mathrm{a}} E_{\text {tot }}$ : electronic energy. ${ }^{\mathrm{b}} \mathrm{ZPE}$ : zero point energy, unscaled

${ }^{\mathrm{c}}$ Imag: imaginary frequency 


\begin{tabular}{l|ccc} 
& \multicolumn{3}{|c}{ trans $24 a ;$ B BLYP/6-31G(d) } \\
\hline $\mathrm{C}$ & 0.00000000 & 0.00000000 & 0.00000000 \\
$\mathrm{O}$ & 1.21878746 & 0.00000000 & 0.00000000 \\
$\mathrm{C}$ & -0.77887544 & 1.36906920 & 0.00000000 \\
$\mathrm{C}$ & -2.03627515 & 1.48484012 & -0.96715810 \\
$\mathrm{C}$ & -1.94539448 & 0.81146397 & -2.36665311 \\
$\mathrm{H}$ & -2.68484957 & 1.30625606 & -3.01394113 \\
$\mathrm{H}$ & -0.96363613 & 1.01755347 & -2.81001853 \\
$\mathrm{C}$ & -2.24844115 & -0.73514465 & -2.40948095 \\
$\mathrm{C}$ & -1.18568258 & 1.74586755 & 1.45331139 \\
$\mathrm{H}$ & -0.46439757 & 1.33676972 & 2.16927715 \\
$\mathrm{H}$ & -1.12592111 & 2.83935355 & 1.51824436 \\
$\mathrm{O}$ & -2.45323881 & 1.26324006 & 1.85601451 \\
$\mathrm{C}$ & -3.56449307 & 1.77923332 & 1.08817536 \\
$\mathrm{C}$ & -3.59063282 & 3.32164294 & 1.08496723 \\
$\mathrm{H}$ & -4.55439542 & 3.66963799 & 0.69803657 \\
$\mathrm{H}$ & -3.48049570 & 3.69753742 & 2.10785060 \\
$\mathrm{H}$ & -2.80900495 & 3.77482424 & 0.46957286 \\
$\mathrm{C}$ & -4.80063332 & 1.28921030 & 1.85181962 \\
$\mathrm{H}$ & -4.84421210 & 1.78426157 & 2.82717467 \\
$\mathrm{H}$ & -5.72020359 & 1.52390739 & 1.30473184 \\
$\mathrm{H}$ & -4.76137046 & 0.21180989 & 2.02732022 \\
$\mathrm{H}$ & -2.00984137 & 2.55880790 & -1.18447164 \\
$\mathrm{C}$ & -3.45770923 & 1.21546508 & -0.36023357 \\
$\mathrm{H}$ & -4.13249682 & 1.81777154 & -0.98787440 \\
$\mathrm{C}$ & -3.97358232 & -0.24159562 & -0.47256040 \\
$\mathrm{H}$ & -5.06569929 & -0.21782050 & -0.37724415 \\
$\mathrm{H}$ & -3.63721940 & -0.83486560 & 0.38095408 \\
$\mathrm{C}$ & -3.64884212 & -0.96316579 & -1.79949887 \\
$\mathrm{H}$ & -4.36667406 & -0.61832637 & -2.55670898 \\
$\mathrm{H}$ & -3.83177825 & -2.03903613 & -1.68146216 \\
$\mathrm{C}$ & -2.22048268 & -1.24455773 & -3.85793370 \\
$\mathrm{H}$ & -1.23774720 & -1.08285485 & -4.31669470 \\
$\mathrm{H}$ & -2.43746232 & -2.31856404 & -3.89860078 \\
$\mathrm{H}$ & -2.96603976 & -0.72669256 & -4.47252568 \\
$\mathrm{H}$ & -0.11735126 & -1.14547203 & -2.18322703 \\
$\mathrm{C}$ & -1.05133304 & -1.21826427 & -1.62073404 \\
$\mathrm{C}$ & -0.97119362 & -1.06472553 & -0.28178428 \\
$\mathrm{H}$ & -1.87258079 & -1.00663643 & 0.30873401 \\
$\mathrm{O}$ & 0.16202969 & 2.33347221 & -0.45332654 \\
$\mathrm{H}$ & 1.03860827 & 1.91622616 & -0.32350124 \\
\hline $\mathrm{E}$ tot ${ }^{\mathrm{a}}$ & & -810.422468787 & \\
$\mathrm{ZPE}$ & & 0.351752 & \\
$\mathrm{Imag}$ & & -- & \\
\hline $\mathrm{E}$ & & &
\end{tabular}

${ }^{\mathrm{a}} E_{\text {tot }}$ : electronic energy. ${ }^{\mathrm{b}} \mathrm{ZPE}$ : zero point energy, unscaled

${ }^{\mathrm{c}}$ Imag: imaginary frequency 


\begin{tabular}{l|ccc} 
& \multicolumn{3}{|c}{ trans $24 \mathbf{b}$; B3LYP/6-31G(d) } \\
\hline $\mathrm{C}$ & 0.00000000 & 0.00000000 & 0.00000000 \\
$\mathrm{O}$ & 1.21059359 & 0.00000000 & 0.00000000 \\
$\mathrm{C}$ & -0.87341397 & 1.32901559 & 0.00000000 \\
$\mathrm{C}$ & -1.98821730 & 1.46920676 & 1.11601620 \\
$\mathrm{C}$ & -2.99351609 & 0.29913364 & 1.37850546 \\
$\mathrm{H}$ & -3.82172147 & 0.73321527 & 1.95768845 \\
$\mathrm{H}$ & -3.43789171 & -0.04011987 & 0.43776042 \\
$\mathrm{C}$ & -2.42537354 & -0.90374271 & 2.20084096 \\
$\mathrm{C}$ & 0.04627021 & 2.56052078 & 0.05994638 \\
$\mathrm{H}$ & 0.93599367 & 2.38272689 & -0.55108516 \\
$\mathrm{H}$ & -0.50815573 & 3.41097837 & -0.36202798 \\
$\mathrm{O}$ & 0.52766436 & 2.83626026 & 1.35876051 \\
$\mathrm{C}$ & -0.48135557 & 3.18210166 & 2.32824275 \\
$\mathrm{C}$ & -1.23224771 & 4.46680771 & 1.91909076 \\
$\mathrm{H}$ & -1.82432655 & 4.83668176 & 2.76326943 \\
$\mathrm{H}$ & -0.51001576 & 5.24240172 & 1.64304110 \\
$\mathrm{H}$ & -1.91728653 & 4.32512149 & 1.07810352 \\
$\mathrm{C}$ & 0.32527305 & 3.48385698 & 3.59648227 \\
$\mathrm{H}$ & 0.93401780 & 4.37943173 & 3.43597862 \\
$\mathrm{H}$ & -0.33475191 & 3.66429660 & 4.45216234 \\
$\mathrm{H}$ & 1.00138425 & 2.66017488 & 3.83753155 \\
$\mathrm{H}$ & -2.60312821 & 2.26872582 & 0.68273346 \\
$\mathrm{C}$ & -1.47280787 & 1.98983158 & 2.49484150 \\
$\mathrm{H}$ & -2.36422150 & 2.39422035 & 2.99947780 \\
$\mathrm{C}$ & -0.94710671 & 0.85114841 & 3.39487853 \\
$\mathrm{H}$ & -0.73454806 & 1.24717811 & 4.39345186 \\
$\mathrm{H}$ & 0.01113176 & 0.48957600 & 3.00409435 \\
$\mathrm{C}$ & -1.94822494 & -0.31327569 & 3.55330873 \\
$\mathrm{H}$ & -2.83124879 & 0.04128087 & 4.10441018 \\
$\mathrm{H}$ & -1.49517075 & -1.10566099 & 4.16474541 \\
$\mathrm{C}$ & -3.48644464 & -1.99282233 & 2.40944447 \\
$\mathrm{H}$ & -3.81043813 & -2.41460238 & 1.45101769 \\
$\mathrm{H}$ & -3.09226116 & -2.81300943 & 3.02084928 \\
$\mathrm{H}$ & -4.37092004 & -1.58740467 & 2.91670445 \\
$\mathrm{H}$ & -1.89078345 & -0.91614100 & -0.41861731 \\
$\mathrm{C}$ & -0.99963170 & -1.07948978 & 0.17756517 \\
$\mathrm{C}$ & -1.15417547 & -1.36058570 & 1.48622048 \\
$\mathrm{H}$ & -0.25821339 & -1.45579267 & 2.09938596 \\
$\mathrm{O}$ & -1.60033605 & 1.34050540 & -1.24310915 \\
$\mathrm{H}$ & -0.95083402 & 1.35048368 & -1.96641213 \\
\hline $\mathrm{E}_{\text {tot }}{ }^{\mathrm{a}}$ & & -810.417876015 & \\
$\mathrm{ZPE}{ }^{\mathrm{b}}$ & & 0.350980 & \\
$\mathrm{Imag}$ & & -- & \\
\hline $\mathrm{E}$ & & &
\end{tabular}

${ }^{\mathrm{a}} E_{\text {tot }}$ : electronic energy. ${ }^{\mathrm{b}} \mathrm{ZPE}$ : zero point energy, unscaled

${ }^{\mathrm{c}}$ Imag: imaginary frequency 


\begin{tabular}{|c|c|c|c|}
\hline & \multicolumn{3}{|c|}{ 26a; UB3LYP/6-31G(d) } \\
\hline C & 0.00000000 & 0.00000000 & 0.00000000 \\
\hline $\mathrm{H}$ & 1.09492576 & 0.00000000 & 0.00000000 \\
\hline C & -0.62115438 & 1.31638939 & 0.00000000 \\
\hline $\mathrm{H}$ & -0.81092710 & 1.83292511 & 0.94273978 \\
\hline $\mathrm{C}$ & -0.96058881 & 2.09642372 & -1.16831218 \\
\hline $\mathrm{O}$ & -1.18447316 & 3.31026159 & -1.04527462 \\
\hline C & -0.98822920 & 1.47420337 & -2.59718627 \\
\hline $\mathrm{C}$ & -1.36124727 & -0.01910725 & -2.79606155 \\
\hline $\mathrm{C}$ & -0.45496952 & -1.08267237 & -2.15075093 \\
\hline $\mathrm{H}$ & -0.70512242 & -2.04873017 & -2.61640177 \\
\hline $\mathrm{H}$ & 0.59250404 & -0.87669059 & -2.39639471 \\
\hline $\mathrm{C}$ & -0.62136659 & -1.23433067 & -0.61202122 \\
\hline C & -1.98170417 & 2.28118857 & -3.45164614 \\
\hline $\mathrm{H}$ & -1.81776681 & 3.35087111 & -3.30805399 \\
\hline $\mathrm{H}$ & -1.80956230 & 2.03194857 & -4.50837259 \\
\hline $\mathrm{O}$ & -3.32082184 & 2.04135509 & -3.05216228 \\
\hline $\mathrm{C}$ & -3.79383103 & 0.68964872 & -3.22408005 \\
\hline $\mathrm{C}$ & -3.88705320 & 0.33394134 & -4.72268171 \\
\hline $\mathrm{H}$ & -4.43535639 & -0.60550239 & -4.85421675 \\
\hline $\mathrm{H}$ & -4.42890005 & 1.12427217 & -5.25237934 \\
\hline $\mathrm{H}$ & -2.91115045 & 0.21301367 & -5.20223024 \\
\hline $\mathrm{C}$ & -5.21289233 & 0.72517815 & -2.64679564 \\
\hline $\mathrm{H}$ & -5.83912393 & 1.38368170 & -3.25714924 \\
\hline $\mathrm{H}$ & -5.66329136 & -0.27365302 & -2.64323939 \\
\hline $\mathrm{H}$ & -5.21178363 & 1.11692390 & -1.62665843 \\
\hline $\mathrm{H}$ & -1.22015971 & -0.14939735 & -3.87707190 \\
\hline C & -2.85399370 & -0.30754087 & -2.47601799 \\
\hline $\mathrm{H}$ & -3.06549622 & -1.30593246 & -2.89011253 \\
\hline $\mathrm{C}$ & -3.08397908 & -0.40859970 & -0.95408694 \\
\hline $\mathrm{H}$ & -4.10838011 & -0.74448437 & -0.75734289 \\
\hline $\mathrm{H}$ & -3.00057742 & 0.58033297 & -0.48930833 \\
\hline C & -2.12502614 & -1.41082396 & -0.28395017 \\
\hline $\mathrm{H}$ & -2.40891957 & -2.42173917 & -0.61117396 \\
\hline $\mathrm{H}$ & -2.26730344 & -1.39103809 & 0.80426461 \\
\hline C & 0.15690570 & -2.47334208 & -0.13027106 \\
\hline $\mathrm{H}$ & 1.22563373 & -2.38176781 & -0.35883707 \\
\hline $\mathrm{H}$ & 0.05443987 & -2.60448131 & 0.95308823 \\
\hline $\mathrm{H}$ & -0.21443233 & -3.38120542 & -0.62002111 \\
\hline $\mathrm{O}$ & 0.34394493 & 1.62121793 & -3.11931970 \\
\hline $\mathrm{H}$ & 0.57384280 & 2.56339875 & -3.04694880 \\
\hline $\mathrm{E}_{\mathrm{tot}}^{\mathrm{a}}{ }^{\mathrm{a}}$ & & 10.391055811 & \\
\hline $\mathrm{ZPE}^{\mathrm{b}}$ & & & \\
\hline Imag & & & \\
\hline
\end{tabular}

${ }^{\mathrm{a}} E_{\text {tot }}$ : electronic energy. ${ }^{\mathrm{b}} \mathrm{ZPE}$ : zero point energy, unscaled

${ }^{\mathrm{c}}$ Imag: imaginary frequency 


\begin{tabular}{l|ccc} 
& \multicolumn{3}{|c}{$\mathbf{2 6 b}$; UB3LYP/6-31G(d) } \\
\hline $\mathrm{C}$ & 0.00000000 & 0.00000000 & 0.00000000 \\
$\mathrm{H}$ & 1.08986000 & 0.00000000 & 0.00000000 \\
$\mathrm{C}$ & -0.69923204 & 1.27390437 & 0.00000000 \\
$\mathrm{H}$ & -0.86564298 & 1.82863762 & -0.92631184 \\
$\mathrm{C}$ & -1.38984620 & 1.81691322 & 1.13872975 \\
$\mathrm{O}$ & -2.03557652 & 2.87626419 & 1.02894426 \\
$\mathrm{C}$ & -1.41145534 & 1.10844860 & 2.52260587 \\
$\mathrm{C}$ & -1.55942851 & -0.46168432 & 2.52135054 \\
$\mathrm{C}$ & -1.94261083 & -1.07168654 & 1.15618604 \\
$\mathrm{H}$ & -2.42142373 & -2.04437722 & 1.33525193 \\
$\mathrm{H}$ & -2.70250385 & -0.44528822 & 0.67088292 \\
$\mathrm{C}$ & -0.73791553 & -1.30881660 & 0.21265055 \\
$\mathrm{C}$ & -0.16817762 & 1.65481210 & 3.33450869 \\
$\mathrm{H}$ & 0.45220122 & 2.30737608 & 2.70953385 \\
$\mathrm{H}$ & -0.60604483 & 2.27441474 & 4.12893013 \\
$\mathrm{O}$ & 0.73512672 & 0.70987528 & 3.86865103 \\
$\mathrm{C}$ & 0.15542213 & -0.49156110 & 4.41645897 \\
$\mathrm{C}$ & -0.90539365 & -0.17688477 & 5.48761047 \\
$\mathrm{H}$ & -1.28211324 & -1.10926742 & 5.92411445 \\
$\mathrm{H}$ & -0.45094309 & 0.41214171 & 6.29189439 \\
$\mathrm{H}$ & -1.75847786 & 0.38369175 & 5.09906573 \\
$\mathrm{C}$ & 1.33279905 & -1.20673785 & 5.08980141 \\
$\mathrm{H}$ & 1.65649880 & -0.63752740 & 5.96697528 \\
$\mathrm{H}$ & 1.04101639 & -2.21066240 & 5.41848610 \\
$\mathrm{H}$ & 2.18777637 & -1.29284537 & 4.41547521 \\
$\mathrm{H}$ & -2.44374639 & -0.58373770 & 3.15199950 \\
$\mathrm{C}$ & -0.42726482 & -1.28480756 & 3.21475754 \\
$\mathrm{H}$ & -0.92176251 & -2.18525597 & 3.61001147 \\
$\mathrm{C}$ & 0.69858694 & -1.76127793 & 2.26851563 \\
$\mathrm{H}$ & 1.27938552 & -2.54519566 & 2.76743888 \\
$\mathrm{H}$ & 1.38973379 & -0.92840817 & 2.09817160 \\
$\mathrm{C}$ & 0.20595401 & -2.30151103 & 0.92047128 \\
$\mathrm{H}$ & -0.33179087 & -3.25001235 & 1.06252536 \\
$\mathrm{H}$ & 1.06746060 & -2.52555272 & 0.27606507 \\
$\mathrm{C}$ & -1.22637447 & -1.88759446 & -1.13384245 \\
$\mathrm{H}$ & -1.89923506 & -1.18952436 & -1.64509581 \\
$\mathrm{H}$ & -0.38295517 & -2.09677635 & -1.80165808 \\
$\mathrm{H}$ & -1.77288987 & -2.82564975 & -0.97288025 \\
$\mathrm{O}$ & -2.59925817 & 1.58570367 & 3.15796440 \\
$\mathrm{H}$ & -2.79310818 & 2.44076465 & 2.72055249 \\
\hline $\mathrm{E}_{\text {tot }}{ }^{\mathrm{a}}$ & & -810.388281129 & \\
$\mathrm{ZPE}$ & & 0.347805 & \\
$\mathrm{Imag}$ & & -- & \\
\hline $\mathrm{d}$ & & & \\
$\mathrm{E}$ & & &
\end{tabular}

${ }^{\mathrm{a}} E_{\text {tot }}$ : electronic energy. ${ }^{\mathrm{b}} \mathrm{ZPE}$ : zero point energy, unscaled

${ }^{\mathrm{c}}$ Imag: imaginary frequency 


\begin{tabular}{l|ccc} 
& \multicolumn{3}{|c}{ acrolein (triplet); UB3LYP/6-31G(d) } \\
\hline $\mathrm{C}$ & 0.00000000 & 0.00000000 & 0.00000000 \\
$\mathrm{H}$ & 1.08835917 & 0.00000000 & 0.00000000 \\
$\mathrm{C}$ & -0.75672338 & 1.24160424 & 0.00000000 \\
$\mathrm{H}$ & -1.00254493 & 1.73782178 & 0.94288608 \\
$\mathrm{C}$ & -1.23433047 & 1.90873654 & -1.18181338 \\
$\mathrm{O}$ & -1.87520859 & 2.96525192 & -1.14229267 \\
$\mathrm{H}$ & -0.99766302 & 1.42425901 & -2.14929028 \\
$\mathrm{H}$ & -0.49451431 & -0.96477152 & 0.09579488 \\
\hline $\mathrm{E}_{\text {tot }}{ }^{\mathrm{a}}$ & \multicolumn{3}{|c}{-191.820505650} \\
$\mathrm{ZPE}^{\mathrm{b}}$ & \multicolumn{3}{|c}{0.057022} \\
$\mathrm{Imag}^{\mathrm{Im}}$ & -- \\
\hline
\end{tabular}

${ }^{\mathrm{a}} E_{\text {tot }}$ : electronic energy. ${ }^{\mathrm{b}} \mathrm{ZPE}$ : zero point energy, unscaled

${ }^{\mathrm{c}}$ Imag: imaginary frequency

\begin{tabular}{l|ccc} 
& \multicolumn{3}{|c}{ cycloheptenone (triplet); UB3LYP/6-31G(d) } \\
\hline $\mathrm{C}$ & 0.00000000 & 0.00000000 & 0.00000000 \\
$\mathrm{H}$ & 1.09082967 & 0.00000000 & 0.00000000 \\
$\mathrm{C}$ & -0.68170460 & 1.28904475 & 0.00000000 \\
$\mathrm{H}$ & -0.91383483 & 1.79647065 & 0.93859561 \\
$\mathrm{C}$ & -1.15552388 & 1.98834909 & -1.17625778 \\
$\mathrm{O}$ & -1.67499886 & 3.10864542 & -1.05105615 \\
$\mathrm{C}$ & -1.09266061 & 1.36055914 & -2.57608028 \\
$\mathrm{H}$ & -2.07213961 & 1.58267455 & -3.01786370 \\
$\mathrm{H}$ & -0.37348878 & 1.95019272 & -3.16144390 \\
$\mathrm{C}$ & -0.78849055 & -0.14488628 & -2.73467444 \\
$\mathrm{H}$ & -1.07504866 & -0.42313624 & -3.75635359 \\
$\mathrm{H}$ & 0.29202560 & -0.32701017 & -2.66850167 \\
$\mathrm{C}$ & -1.52054909 & -1.05545812 & -1.72661950 \\
$\mathrm{H}$ & -1.71263813 & -2.03993782 & -2.17033610 \\
$\mathrm{H}$ & -2.50507594 & -0.62591598 & -1.49835380 \\
$\mathrm{C}$ & -0.71828243 & -1.25697322 & -0.41031829 \\
$\mathrm{H}$ & -1.40599414 & -1.59904159 & 0.37884574 \\
$\mathrm{H}$ & 0.02032745 & -2.05554435 & -0.54655431 \\
\hline $\mathrm{E}_{\text {tot }}^{\mathrm{a}}$ & \multicolumn{3}{|c}{-347.876600755} \\
$\mathrm{ZPE}$ & 0.152605 \\
Imag & \multicolumn{3}{|c}{-} \\
\hline${ }^{\mathrm{a}} \mathrm{E}_{\text {tot }}$ : electronic energy. ${ }^{\mathrm{b}}$ ZPE: zero point energy, unscaled \\
${ }^{\mathrm{c}}$ Imag: imaginary frequency
\end{tabular}




\section{Complete Reference 44}

(44) Frisch, M. J.; Trucks, G. W.; Schlegel, H. B;. Scuseria, G. E.; Robb, M. A.; Cheeseman, J. R.; Zakrzewski, V. G.; Montgomery Jr., J. A.; Stratmann, R. E.; Burant, J. C.; Dapprich, S.; Millam, J. M.; Daniels, A. D.; Kudin, K.N.; Strain, M. C; Farkas, O.; Tomasi, J.; Barone, V.; Cossi, M.; Cammi, R.; Mennucci, B.; Pomelli, C.; Adamo, C.; Clifford, S.; Ochterski, J.; Petersson, G. A.; Ayala, P. Y.; Cui, Q.; Morokuma, K.; Salvador, P.; Dannenberg, J. J.; Malick, D. K.; Rabuck, A. D.; Raghavachari, K.; Foresman, J. B.; Cioslowski, J.; Ortiz, J. V.; Baboul, A. G.; Stefanov, B. B.; Liu, G.; Liashenko, A.; Piskorz, P.; Komaromi, I.; Gomperts, R.; Martin, R. L.; Fox, D. J.; Keith, T.; Al-Laham, M. A.; Peng, C. Y.; Nanayakkara, A.; Challacombe, M.; Gill, P. M. W.; Johnson, B.; Chen, W.; Wong, M. W.; Andres, J. L.; Gonzalez, C.; Head-Gordon M.; Replogle, E. S.; and Pople, J. A.; Gaussian 98, Revision A.11; Gaussian Inc.: Pittsburgh, PA, 2001. 


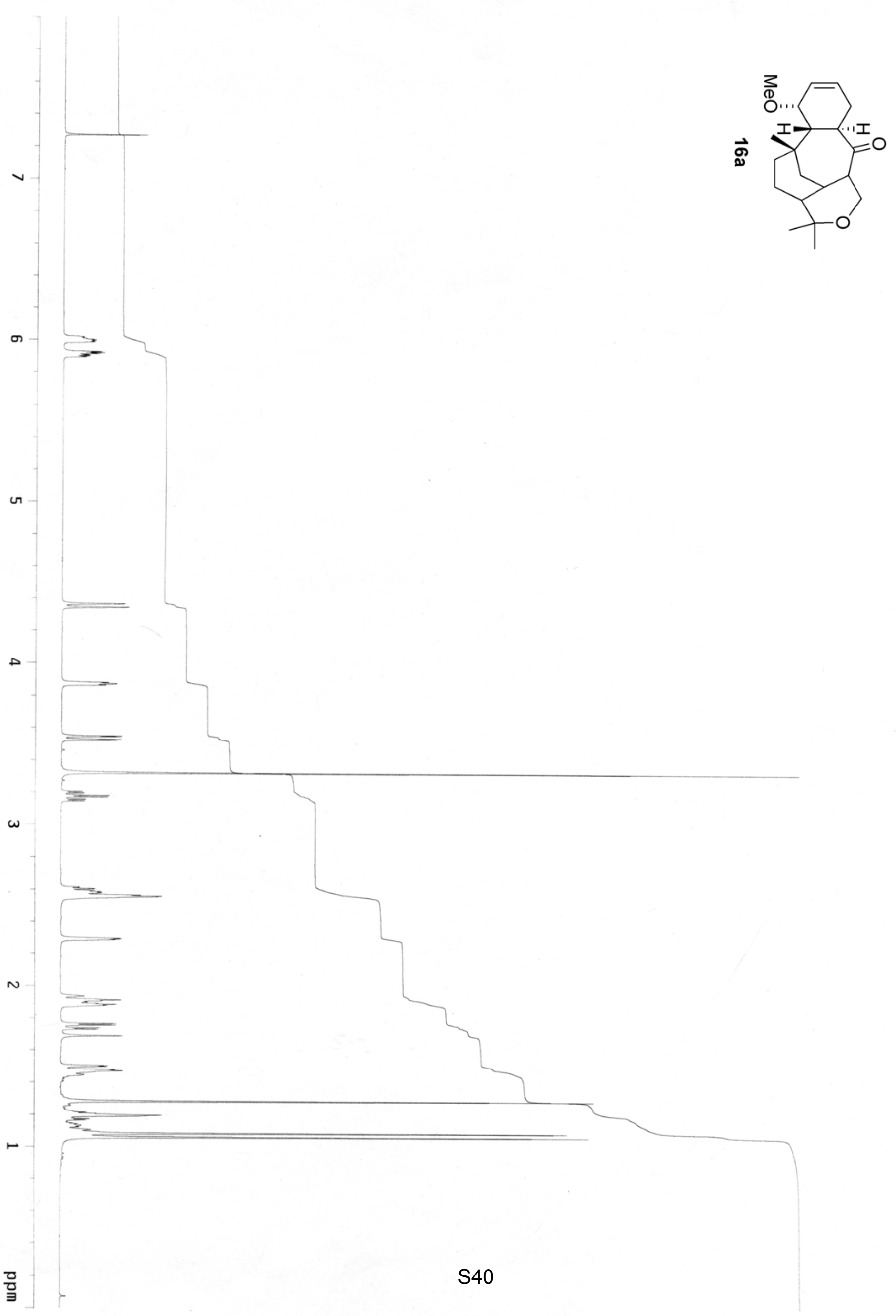




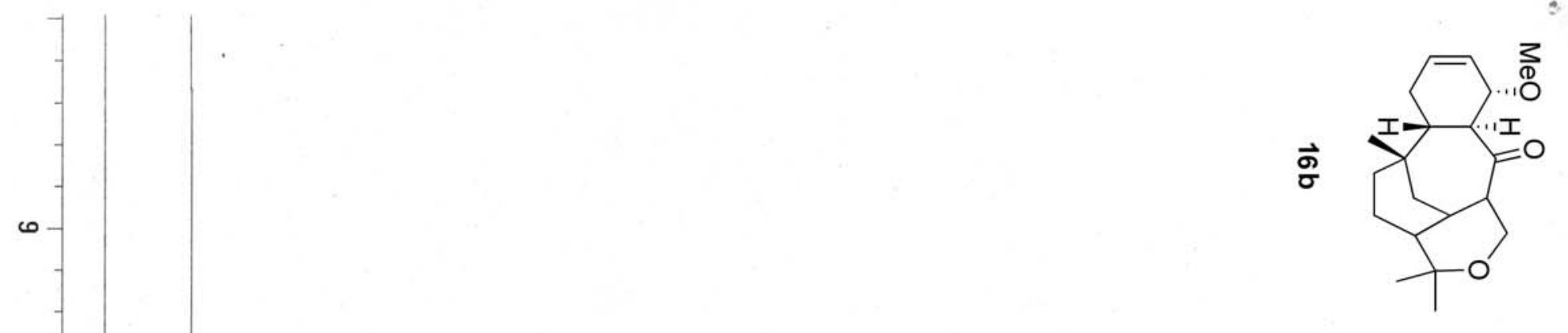

$\infty$

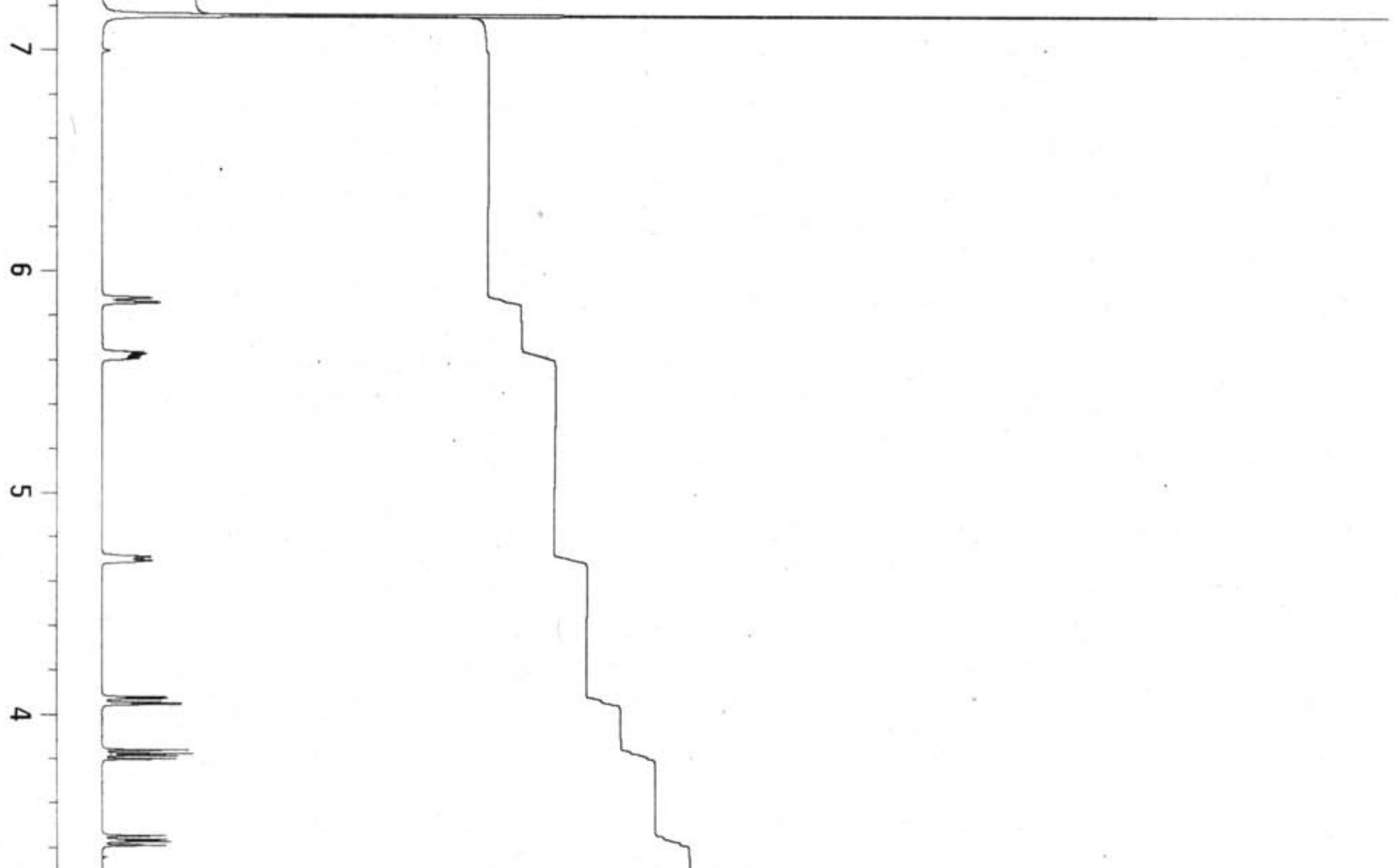

$\omega$

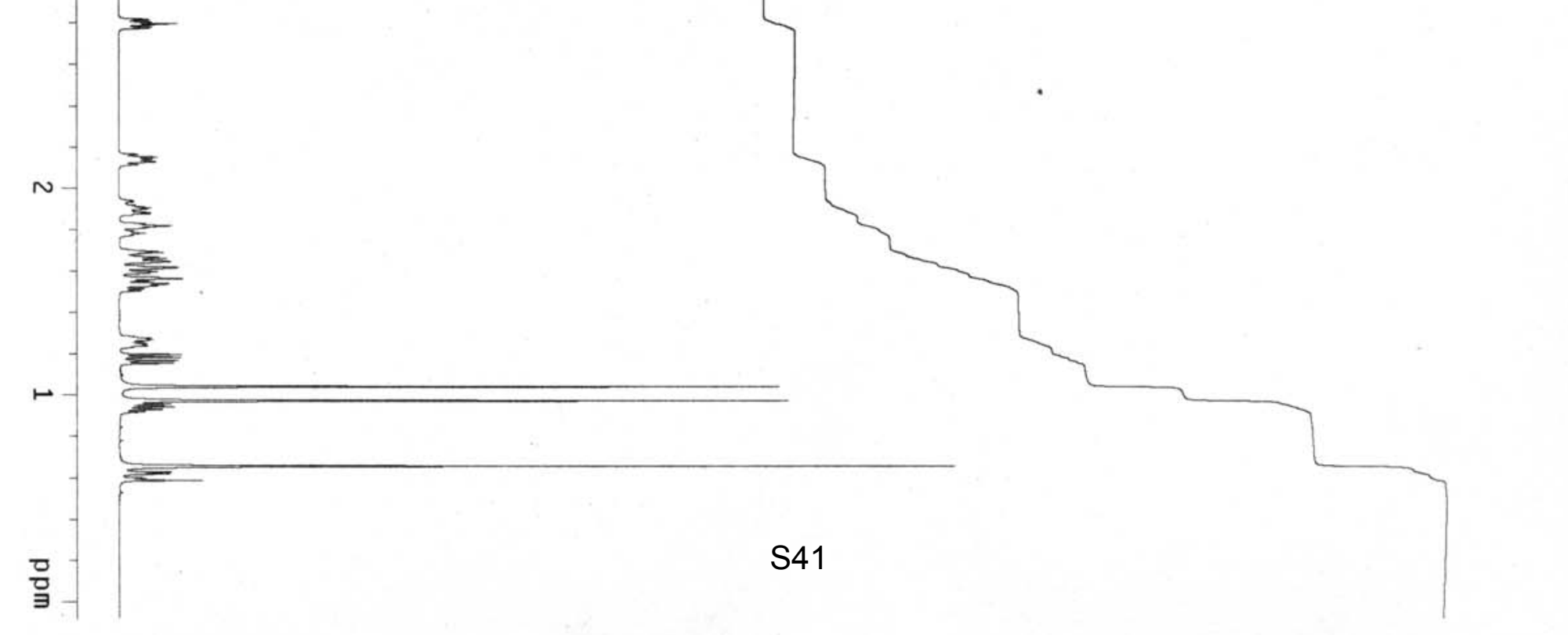




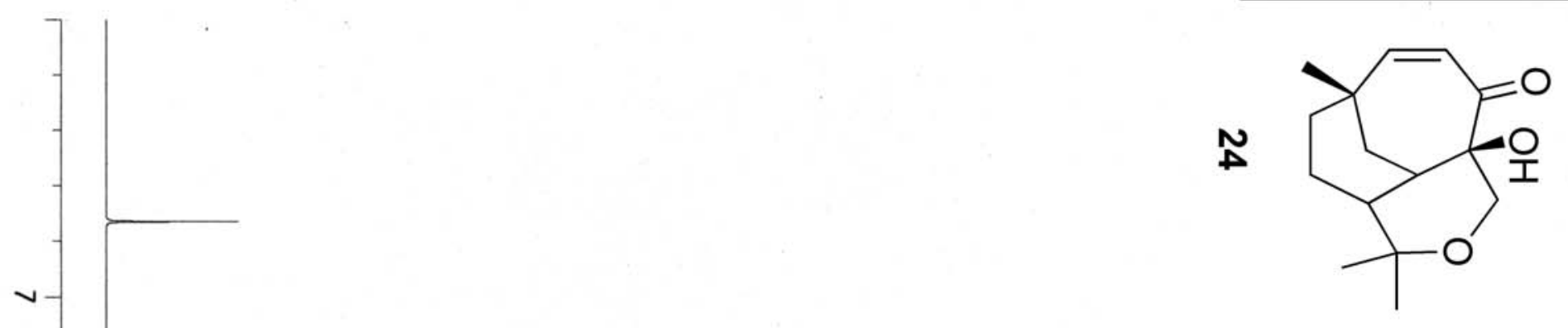

m

ज

$\triangle$

$\omega$



N

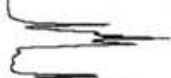

$=$

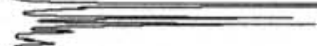

 


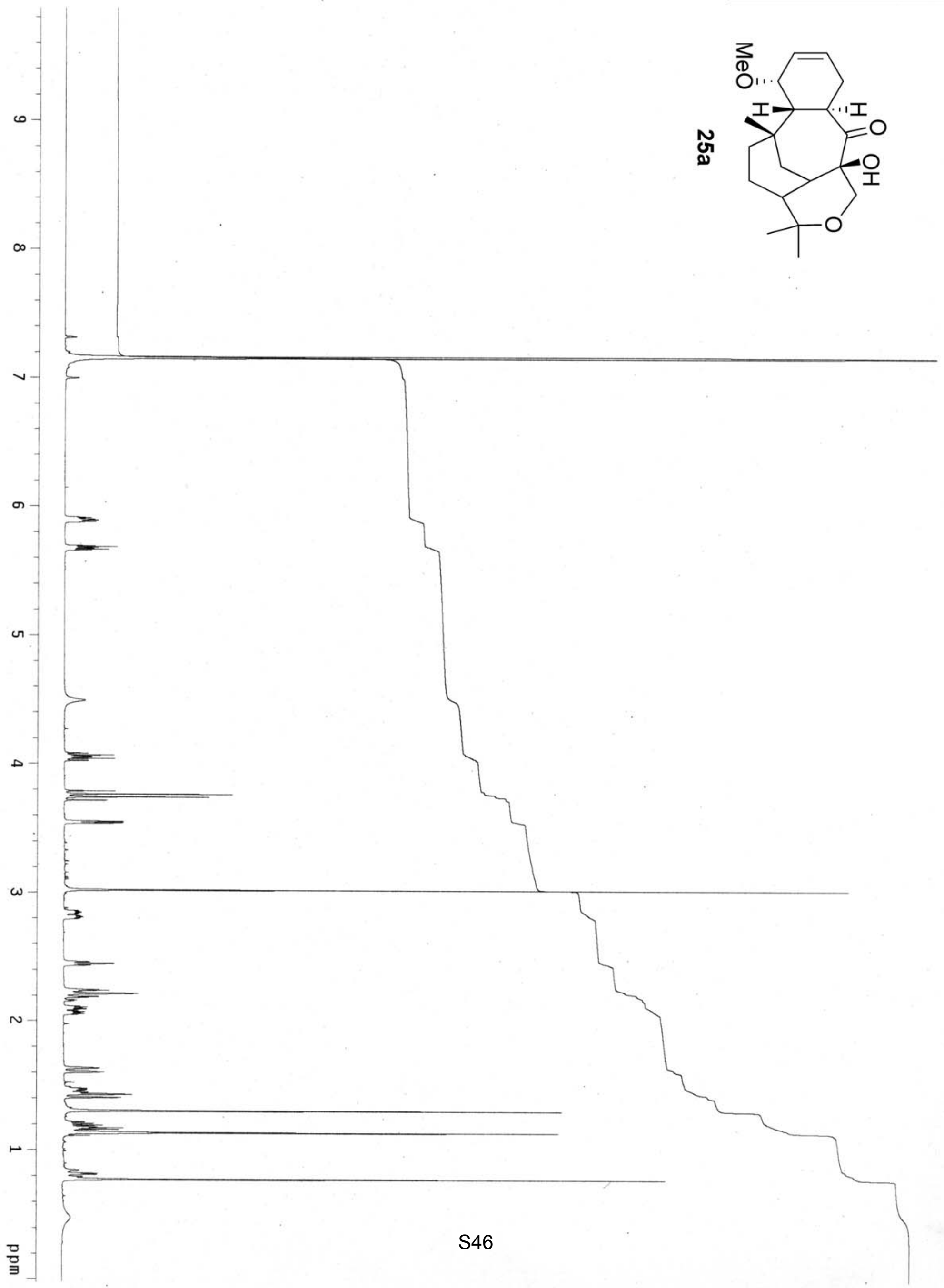




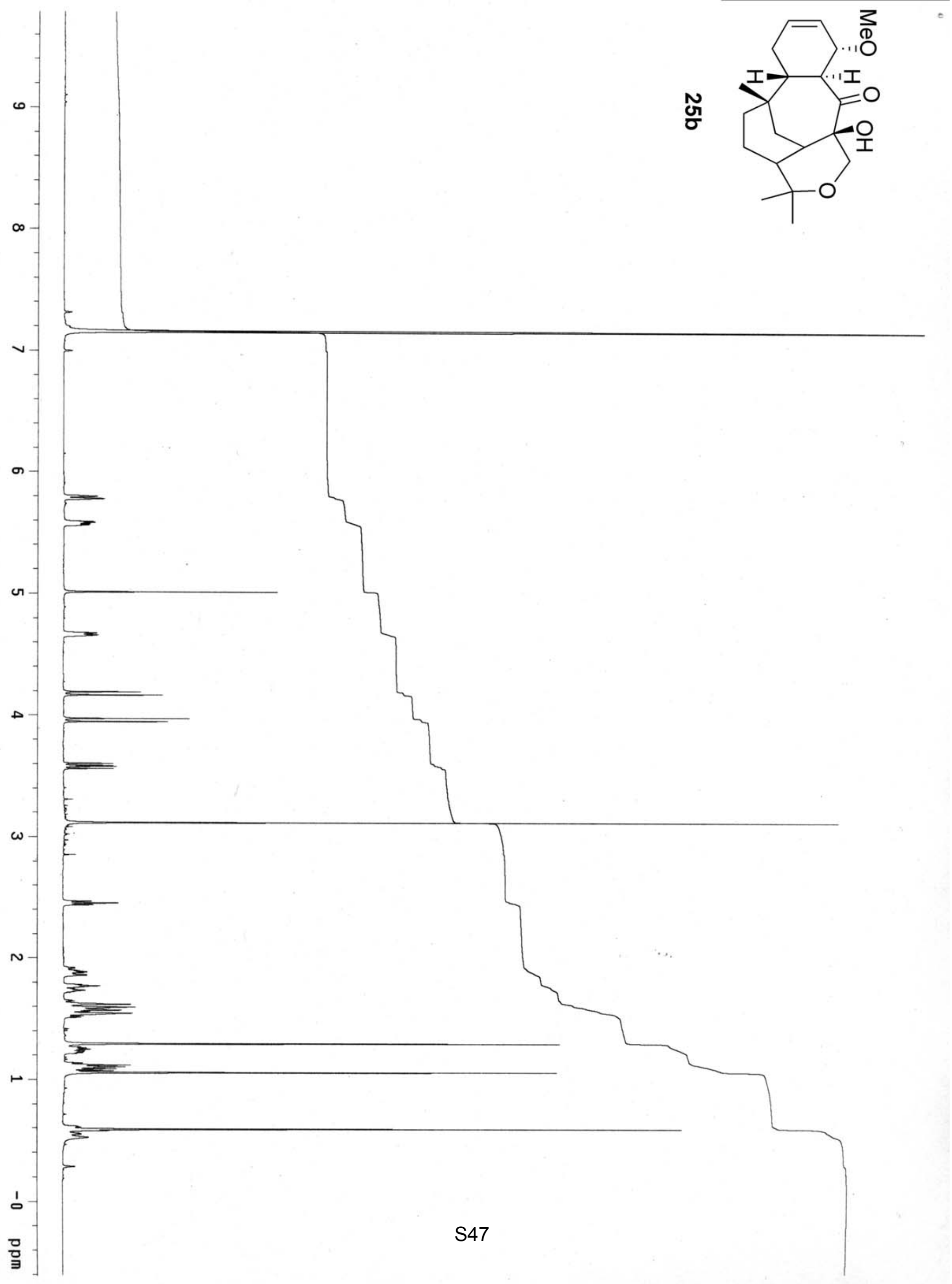




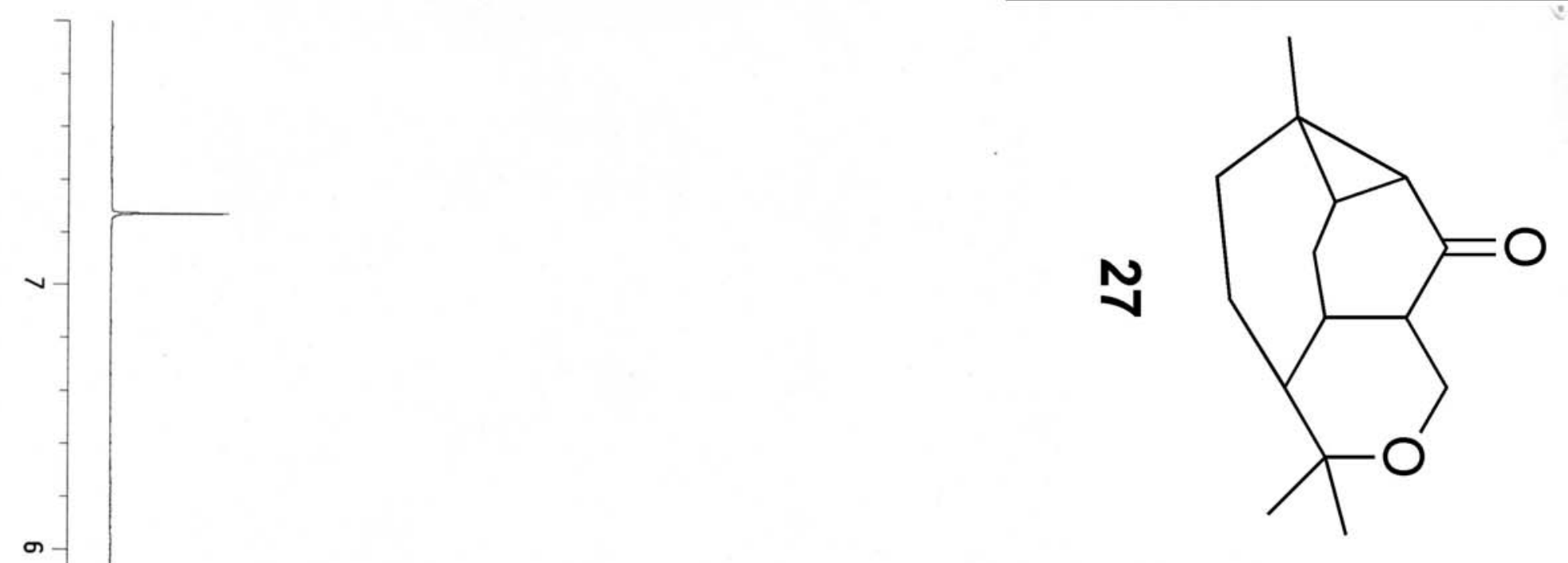

